ESAIM: M2AN 46 (2012) 1029-1054

DOI: $10.1051 / \mathrm{m} 2 \mathrm{an} / 2011069$
ESAIM: Mathematical Modelling and Numerical Analysis

www.esaim-m2an.org

\title{
MODELLING AND SIMULATION OF LIQUID-VAPOR PHASE TRANSITION IN COMPRESSIBLE FLOWS BASED ON THERMODYNAMICAL EQUILIBRIUM*
}

\author{
Gloria Faccanoni ${ }^{1}$, Samuel KokH $^{2}$ And Grégoire Allaire ${ }^{3,4}$
}

\begin{abstract}
In the present work we investigate the numerical simulation of liquid-vapor phase change in compressible flows. Each phase is modeled as a compressible fluid equipped with its own equation of state (EOS). We suppose that inter-phase equilibrium processes in the medium operate at a short time-scale compared to the other physical phenomena such as convection or thermal diffusion. This assumption provides an implicit definition of an equilibrium EOS for the two-phase medium. Within this framework, mass transfer is the result of local and instantaneous equilibria between both phases. The overall model is strictly hyperbolic. We examine properties of the equilibrium EOS and we propose a discretization strategy based on a finite-volume relaxation method. This method allows to cope with the implicit definition of the equilibrium EOS, even when the model involves complex EOS's for the pure phases. We present two-dimensional numerical simulations that shows that the model is able to reproduce mechanism such as phase disappearance and nucleation.
\end{abstract}

Mathematics Subject Classification. 76T10, 76N10, 65M08.

Received January 24, 2011. Revised July 13, 2011.

Published online February 13, 2012.

\section{INTRODUCTION}

The simulation of liquid-vapor phase change phenomena in fluid flows raises challenging problems pertaining to physical modeling, mathematical analysis and numerical analysis. It is all the more important that it concerns many industrial applications. For example, the prediction of the boiling crisis is a crucial problem for the safety studies in the nuclear industry $[14-16,18,46,48,54]$. In the present article, we focus on a model of phase change for compressible two-phase flows (away from the critical point) and propose a numerical algorithm for solving it.

Keywords and phrases. Compressible flows, two-phase flows, hyperbolic systems, phase change, relaxation method.

* This work has been achieved within the framework of the NEPTUNE project, financially supported by CEA (Commissariat à l'Énergie Atomique), EDF (Électricité de France), IRSN (Institut de Radioprotection et de Sûreté Nucléaire) and AREVA-NP. The authors thank the CEA for its financial support. Grégoire Allaire is a member of the DEFI project at INRIA Saclay Ile-de-France and is partially supported by the Chair "Mathematical modeling and numerical simulation", F-EADS - École Polytechnique - INRIA.

1 IMATH - Université du Sud Toulon-Var, Avenue de l'Université, 83957 La Garde, France. faccanon@univ-tln.fr

2 DEN/DANS/DM2S/SFME/LETR, Commissariat à l'Énergie Atomique Saclay, 91191 Gif-sur-Yvette, France. samuel.kokh@cea.fr

3 Conseiller Scientifique du DM2S - Commissariat à l'Énergie Atomique Saclay, 91191 Gif-sur-Yvette, France.

4 CMAP, École Polytechnique, CNRS, 91128 Palaiseau, France. allaire@cmap.polytechnique.fr 
A classic approach consists in modeling the two-phase system by a single fluid with a Van der Waals type equation of state (EOS) [22]. Far from the critical point, each phase corresponds to a stable branch of the EOS, which are connected by the spinodal zone. The intrinsic instability of the fluid in the spinodal zone requires to account for very small scale effects by the means of Korteweg-type tensors (see $[6,24,27,33,37,52]$ and the references therein) including dissipative effects as well as dispersive effects. In other words, the interface between the two phases is implicitly modeled as a thick transition zone. A drawback of such models is that they call for very fine grids when discretizing the system.

It is possible to model the interface as a discontinuity locus connecting two pure phase states. Such discontinuity can be obtained by taking the limit in the Korteweg model when the interface thickness tends to zero $[10,17,38,51,53]$. However this requires the knowledge of the whole EOS for the two-phase material including the spinodal zone. Unfortunately, experimental data for the spinodal zone are often not available.

We follow here an alternate approach that has been examined by several authors for the past years $[2,4,10,11,18,19,21,23,25,29,31,34,45,48]$. The key idea lies in avoiding the spinodal zone by assuming that the driving physical phenomena are kinetical effects that act on both phases at a short time scale compared to the hydrodynamics and thermal diffusion in agreement with classic thermodynamics. This comes down to assuming that each phase is modeled by a compressible fluid and that the EOS of the two-phase system is obtained by a convexification procedure (see Prop. 4.2). We note that this construction is more general than the classic convexification procedure based on the Maxwell rule of a Van der Waals EOS $[9,28,34,45]$ : in the present model each pure phase is equipped independently with its own EOS. If we consider the particular case when each pure phase EOS matches a stable branch of the Van der Waals EOS, the resulting equilibrium EOS of our model coincides with the classic Maxwell construction for the Van der Waals EOS [30]. For all other cases we propose a simple way to compute the equilibrium EOS, based on solving a single nonlinear equation that we call "phase-change equation". We demonstrate its usefulness in the case of pure phases having a stiffened gas EOS.

Within this context, the mass transfer between both phases is driven by instantaneous equilibria in the composition of the two-phase medium with respect to phasic pressures, temperatures and Gibbs potentials. An important issue is that, as it is expected, the resulting EOS does no longer fulfill classic strict convexity thermodynamics hypotheses for the entropy. Moreover, the process of reaching equilibria (and equivalently the convexification process) involves solving a nonlinear system that combines the EOS of the pure phases. Nevertheless, it is possible to show that, under simple hypotheses on the pure phase EOSs, the system is strictly hyperbolic (Thm. 5.2). This property, announced in [2], is important for the theoretical and numerical resolution of the system.

Once this well-posedness property is obtained, we can use the relaxation framework of $[7,12,13,35,43]$ to interpret the equilibria between both phases (and equivalently the convexification process) as the limit of a relaxation procedure. This modeling interpretation provides means of discretizing the system thanks to a twostep relaxation strategy.

This paper is structured as follows: in Section 2 we present the overall structure of our model. In Section 3 we first recall basic thermodynamics principles for pure phases, then we provide our system with an off-equilibrium entropy. Then we introduce the equilibrium entropy of the system. In Section 4 we detail properties of this entropy law related to the geometrical construction of its graph, convexification principles of the pure phases entropy and convexity properties. The definition of this equilibrium law involves the resolution of a system of nonlinear equations. We show that it is possible to reduce this system to a nonlinear scalar equation by using classic thermodynamical state laws for saturated states. We recall in Section 5 key well-posedness property of this system. In Section 6 we show that the system can be interpreted by means of a relaxation approach to other two-phase system. We propose in Section 7 a multi-step relaxation discretization strategy that allows to decouple computation of the equilibrium thermodynamical parameters from the approximation of the convection by confining it in the projection step. Finally in Section 8 we present two-dimensional numerical simulations of boiling-type tests. 


\section{Model Structure}

In the sequel the subscript $\alpha=l$ (resp. $\alpha=v$ ) will refer to the liquid (resp. vapor) phase. Let $\tau_{\alpha}$ and $\varepsilon_{\alpha}$ denote respectively the specific volume and the specific internal energy of the phase $\alpha=l, v$. We define the cone of admissible states

$$
\mathcal{C} \stackrel{\text { def }}{=}\{(\tau, \varepsilon) \quad \mid \quad \tau>0, \varepsilon>0\}
$$

and $\mathbf{w}_{\alpha} \stackrel{\text { def }}{=}\left(\tau_{\alpha}, \varepsilon_{\alpha}\right)$. In the following we shall always assume that $\mathbf{w}_{\alpha} \in \mathcal{C}$.

We suppose that the fluid will never reach the critical point and therefore each phase can be modelled as a compressible fluid equipped with its complete EOS $\mathbf{w}_{\alpha} \mapsto s_{\alpha}$, where $s_{\alpha}$ denotes the specific entropy of the phase $\alpha=l, v$. The assumption on the pure phase entropy laws will be detailed in Section 3.1.

In order to define the composition of the two-phase media, we introduce three fractions $[2,4,10,11,18,19,21,29,31]$ :

- $y_{l}\left(\right.$ resp. $\left.y_{v}\right)$ the mass fraction of the phase $\alpha=l$ (resp. $\alpha=v$ ); we note $y \stackrel{\text { def }}{=} y_{l}$ and we suppose $y \in[0,1]$ (that implies $y_{v}=1-y$ );

- $z_{l}\left(\right.$ resp. $\left.z_{v}\right)$ the volume fraction of the phase $\alpha=l$ (resp. $\alpha=v$ ); we note $z \stackrel{\text { def }}{=} z_{l}$ and we suppose $z \in[0,1]$ (that implies $z_{v}=1-z$ );

- $\psi_{l}\left(\right.$ resp. $\left.\psi_{v}\right)$ the energy fraction of the phase $\alpha=l$ (resp. $\left.\alpha=v\right)$; we note $\psi \stackrel{\text { def }}{=} \psi_{l}$ and we suppose $\psi \in[0,1]$ (that implies $\psi_{v}=1-\psi$ ).

Let us now define the global specific volume $\tau$ and internal energy $\varepsilon$ of the two-phase medium: for $\mathbf{w} \stackrel{\text { def }}{=}(\tau, \varepsilon)$ $\in \mathcal{C}$, the additivity of the volume and the internal energy implies that

$$
\mathbf{w} \stackrel{\text { def }}{=}\left(\begin{array}{l}
\tau \\
\varepsilon
\end{array}\right)=\sum_{\alpha=l, v} y_{\alpha}\left(\begin{array}{l}
\tau_{\alpha} \\
\varepsilon_{\alpha}
\end{array}\right)=\sum_{\alpha=l, v} y_{\alpha} \mathbf{w}_{\alpha}
$$

We define the set $\mathcal{Q}(\mathbf{w})$ as follows

$$
\mathcal{Q}(\mathbf{w}) \stackrel{\text { def }}{=}\left\{\begin{array}{l|c}
\left(\mathbf{w}_{l}, \mathbf{w}_{v}, y_{l}, y_{v}\right) \in \mathcal{C}^{2} \times[0,1]^{2} & \begin{array}{c}
1=y_{l}+y_{v} \\
\mathbf{w}=y_{l} \mathbf{w}_{l}+y_{v} \mathbf{w}_{v}
\end{array}
\end{array}\right\} .
$$

Therefore

$$
z=y \frac{\tau_{l}}{\tau} \quad \text { and } \quad \psi=y \frac{\varepsilon_{l}}{\varepsilon} .
$$

We suppose that both phases have the same velocity $\mathbf{u}$ and we set $\varrho \stackrel{\text { def }}{=} 1 / \tau$. If we neglect the viscous dissipative effects, then the two-phase medium is governed by the system

$$
\partial_{t} \mathbf{U}+\operatorname{div}\left[\mathbf{F}^{\mathrm{eq}}(\mathbf{U})\right]=\mathbf{S}_{\text {tension }}(\mathbf{U}, z)+\mathbf{S}_{\text {gravity }}(\mathbf{U})-\operatorname{div}\left[\mathbf{Q}_{\text {heat }}(\mathbf{U}, z)\right]
$$

where

$$
\mathbf{U} \stackrel{\text { def }}{=}\left(\begin{array}{c}
\varrho \\
\varrho \mathbf{u} \\
\varrho \varepsilon+\varrho|\mathbf{u}|^{2} / 2
\end{array}\right), \quad \mathbf{F}^{\mathrm{eq}}(\mathbf{U}) \stackrel{\text { def }}{=}\left(\begin{array}{c}
\varrho \mathbf{u} \otimes \mathbf{u}+P^{\mathrm{eq}} \mathrm{Id} \\
\left(\varrho \varepsilon+\varrho|\mathbf{u}|^{2} / 2+P^{\mathrm{eq}}\right) \mathbf{u}
\end{array}\right) .
$$

The terms $\mathbf{S}_{\text {gravity }}(\mathbf{U})$ and $\mathbf{Q}_{\text {heat }}(\mathbf{U}, z)$ that account respectively for gravity and thermal diffusion effects read:

$$
\mathbf{S}_{\text {gravity }}(\mathbf{U}) \stackrel{\text { def }}{=}\left(\begin{array}{c}
0 \\
\varrho \mathbf{g} \\
\varrho \mathbf{g} \cdot \mathbf{u}
\end{array}\right), \quad \mathbf{Q}_{\text {heat }}(\mathbf{U}, z) \stackrel{\text { def }}{=}\left(\begin{array}{c}
0 \\
0 \\
\vartheta \operatorname{grad} T
\end{array}\right)
$$


where $\vartheta \stackrel{\text { def }}{=} z \vartheta_{l}+(1-z) \vartheta_{v}$ is the thermal conductivity of the two-phase medium and $\vartheta_{\alpha}$ is the thermal conductivity of the phase $\alpha$. The surface tension effects are modeled thanks to the continuum surface force (CSF) model of $[8]$ which reads here

$$
\mathbf{S}_{\text {tension }}(\mathbf{U}, z) \stackrel{\text { def }}{=}\left(\begin{array}{c}
0 \\
\mathfrak{T} \\
\mathfrak{T} \cdot \mathbf{u}
\end{array}\right), \quad \mathfrak{T} \stackrel{\text { def }}{=} \xi \frac{\operatorname{grad} z}{|\operatorname{grad} z|} \operatorname{div}\left(\frac{\operatorname{grad} z}{|\operatorname{grad} z|}\right)
$$

where $\xi$ is the capillary coefficient.

The pressure law $(\tau, \varepsilon) \mapsto P^{\mathrm{eq}}(\tau, \varepsilon)$ derives from an equilibrium EOS whose construction will be presented in Section 4. This EOS accounts for the mass transfer between both phases by imposing local and instantaneous thermodynamics equilibria in the composition of the medium.

\section{Equilibrium EOS: DEFINITION}

First we shall recall basic notations and assumptions pertaining to homogeneous pure fluids state laws. Then we will postulate an off-equilibrium state law for a medium composed of a liquid phase and a vapor phase. Finally, we define equilibrium within this medium that will provide us an equilibrium EOS accounting for mass transfer between both phases.

\subsection{Pure phase EOS}

In the following we suppose that $s_{\alpha}$ is a $\mathrm{C}^{2}$ regular function over the cone $\mathcal{C}(2.1)$. We note the first and second derivatives of $s_{\alpha}$ as follows

$$
\begin{aligned}
& \left.\left(s_{\alpha}\right)_{\varepsilon_{\alpha}} \stackrel{\text { def }}{=} \frac{\partial s_{\alpha}}{\partial \varepsilon_{\alpha}}\right|_{\tau_{\alpha}},\left.\quad\left(s_{\alpha}\right)_{\tau_{\alpha}} \quad \stackrel{\text { def }}{=} \frac{\partial s_{\alpha}}{\partial \tau_{\alpha}}\right|_{\varepsilon_{\alpha}}, \\
& \left.\left(s_{\alpha}\right)_{\varepsilon_{\alpha} \varepsilon_{\alpha}} \stackrel{\text { def }}{=} \frac{\partial^{2} s_{\alpha}}{\partial \varepsilon_{\alpha}^{2}}\right|_{\tau_{\alpha}} ^{\alpha},\left.\quad\left(s_{\alpha}\right)_{\tau_{\alpha} \tau_{\alpha}} \stackrel{\text { def }}{=} \frac{\partial^{2} s_{\alpha}}{\partial \tau_{\alpha}^{2}}\right|_{\varepsilon_{\alpha}} ^{\alpha}, \quad\left(s_{\alpha}\right)_{\tau_{\alpha} \varepsilon_{\alpha}} \stackrel{\text { def }}{=} \frac{\partial^{2} s_{\alpha}}{\partial \tau_{\alpha} \partial \varepsilon_{\alpha}}
\end{aligned}
$$

and we note the Hessian matrix of $s_{\alpha}$

$$
\mathrm{d}^{2} s_{\alpha} \stackrel{\text { def }}{=}\left[\begin{array}{ll}
\left(s_{\alpha}\right)_{\tau_{\alpha} \tau_{\alpha}} & \left(s_{\alpha}\right)_{\tau_{\alpha} \varepsilon_{\alpha}} \\
\left(s_{\alpha}\right)_{\tau_{\alpha} \varepsilon_{\alpha}} & \left(s_{\alpha}\right)_{\varepsilon_{\alpha} \varepsilon_{\alpha}}
\end{array}\right] .
$$

The temperature $T_{\alpha}$, the pressure $P_{\alpha}$, the free enthalpy $g_{\alpha}$ and the square of the speed of sound $c_{\alpha}$ are defined classically by

$$
\begin{gathered}
T_{\alpha} \stackrel{\text { def }}{=} \frac{1}{\left(s_{\alpha}\right)_{\varepsilon_{\alpha}}}, \quad P_{\alpha} \stackrel{\text { def }}{=} \frac{\left(s_{\alpha}\right)_{\tau_{\alpha}}}{\left(s_{\alpha}\right)_{\varepsilon_{\alpha}}}, \quad g_{\alpha} \stackrel{\text { def }}{=} \varepsilon_{\alpha}+P_{\alpha} \tau_{\alpha}-T_{\alpha} s_{\alpha}, \\
c_{\alpha}^{2} \stackrel{\text { def }}{=} \tau_{\alpha}^{2}\left(\left.P_{\alpha} \frac{\partial P_{\alpha}}{\partial \varepsilon_{\alpha}}\right|_{\tau_{\alpha}}-\left.\frac{\partial P_{\alpha}}{\partial \tau_{\alpha}}\right|_{\varepsilon_{\alpha}}\right) \\
=-\tau_{\alpha}^{2} T_{\alpha}\left[P_{\alpha},-1\right] \mathrm{d}^{2} s_{\alpha}\left[\begin{array}{c}
P_{\alpha} \\
-1
\end{array}\right] .
\end{gathered}
$$

Thermodynamics characterizes the derivatives of $s_{\alpha}$ : first, temperature positivity requires $s_{\alpha}$ to be a strictly increasing function of $\varepsilon_{\alpha}$; second, a stability assumption is enforced by assuming a definite negative Hessian matrix $\mathrm{d}^{2} s_{\alpha}$ for $s_{\alpha}$. Therefore, for all $\mathbf{w}_{\alpha} \in \mathcal{C}$, we have

$$
\left\{\begin{array}{l}
\left(s_{\alpha}\right)_{\varepsilon_{\alpha}}>0, \\
\left(s_{\alpha}\right)_{\varepsilon_{\alpha} \varepsilon_{\alpha}}\left(s_{\alpha}\right)_{\tau_{\alpha} \tau_{\alpha}}>\left(\left(s_{\alpha}\right)_{\tau_{\alpha} \varepsilon_{\alpha}}\right)^{2}, \\
\left.\left(s_{\alpha}\right)_{\varepsilon_{\alpha} \varepsilon_{\alpha}}<0, \quad \text { (or equivalently }\left(s_{\alpha}\right)_{\tau_{\alpha} \tau_{\alpha}}<0\right) .
\end{array}\right.
$$

Let us note that relations (3.3b)-(3.3c) imply that $\mathbf{w}_{\alpha} \mapsto s_{\alpha}$ is strictly concave but the converse is wrong. 


\subsection{Off-equilibrium two-phase EOS}

Following classic thermodynamics (see $[9,28]$ ), we define an entropy $\sigma$ for the off-equilibrium medium: for all $\left(\mathbf{w}_{l}, \mathbf{w}_{v}, y_{l}, y_{v}\right) \in \mathcal{C}^{2} \times[0,1]^{2}$,

$$
\sigma\left(\mathbf{w}_{l}, \mathbf{w}_{v}, y_{l}, y_{v}\right) \stackrel{\text { def }}{=} y_{l} s_{l}\left(\mathbf{w}_{l}\right)+y_{v} s_{v}\left(\mathbf{w}_{v}\right) .
$$

The entropy $\sigma$ can also be expressed thanks to the variables $(y, z, \psi, \mathbf{w}=(\tau, \varepsilon)) \in[0,1]^{3} \times \mathcal{C}$ as follows (for the sake of simplicity we keep the same notation)

$$
\sigma(y, z, \psi, \tau, \varepsilon)= \begin{cases}s_{l}(\tau, \varepsilon) & \text { if } y=1 \\ y s_{l}\left(\frac{z}{y} \tau, \frac{\psi}{y} \varepsilon\right)+(1-y) s_{v}\left(\frac{1-z}{1-y} \tau, \frac{1-\psi}{1-y} \varepsilon\right) & \text { if } 0<y<1 \\ s_{v}(\tau, \varepsilon) & \text { if } y=0\end{cases}
$$

\subsection{Equilibrium two-phase EOS}

Following the lines of $[9,28,29,31]$, for a given global state $\mathbf{w} \stackrel{\text { def }}{=}(\tau, \varepsilon) \in \mathcal{C}$, we can define the equilibrium composition parameters $\left(\mathbf{w}_{l}^{*}, \mathbf{w}_{v}^{*}, y_{l}^{*}, y_{v}^{*}\right) \in \mathcal{Q}(\mathbf{w})$, in agreement with the second law of Thermodynamics, as maximizer of $\left(\mathbf{w}_{l}, \mathbf{w}_{v}, y_{l}, y_{v}\right) \mapsto \sigma$ and we note $z^{*} \stackrel{\text { def }}{=} y^{*} \frac{\tau_{l}^{*}}{\tau}, \psi^{*} \stackrel{\text { def }}{=} y^{*} \frac{\varepsilon_{l}^{*}}{\varepsilon}$.

We now assume that the fluid always instantaneously reaches equilibrium. We can introduce an equilibrium entropy $\mathbf{w} \mapsto s^{\text {eq }}$ for the two-phase medium by the following definition: for all $\mathbf{w} \in \mathcal{C}$,

$$
s^{\mathrm{eq}}(\mathbf{w})=\sup \left\{\sigma\left(\mathbf{w}_{l}, \mathbf{w}_{v}, y_{l}, y_{v}\right) \quad \mid \quad\left(\mathbf{w}_{l}, \mathbf{w}_{v}, y_{l}, y_{v}\right) \in \mathcal{Q}(\mathbf{w})\right\}
$$

or equivalently

$$
s^{\mathrm{eq}}(\mathbf{w})=\sup \left\{\begin{array}{l|l}
\sigma(y, z, \psi, \mathbf{w}) & \begin{array}{l}
y \in[0,1] \\
z \in[0,1] \\
\psi \in[0,1]
\end{array}
\end{array}\right\} .
$$

For the equilibrium two-phase medium, the pressure $P^{\mathrm{eq}}$, the temperature $T^{\mathrm{eq}}$, the chemical potential $g^{\mathrm{eq}}$ and the speed of sound $c^{\mathrm{eq}}$ are then defined thanks to the classic formulas

$$
\begin{gathered}
P^{\mathrm{eq}} \stackrel{\text { def }}{=} \frac{s_{\tau}^{\mathrm{eq}}}{s_{\varepsilon}^{\mathrm{eq}}}, \quad T^{\mathrm{eq}} \stackrel{\text { def }}{=} \frac{1}{s_{\varepsilon}^{\mathrm{eq}}}, \quad g^{\mathrm{eq}} \stackrel{\text { def }}{=} \varepsilon+P^{\mathrm{eq}} \tau-T^{\mathrm{eq}} s^{\mathrm{eq}}, \\
\left(c^{\mathrm{eq}}\right)^{2}=\tau^{2}\left(\left.P^{\mathrm{eq}} \frac{\partial P^{\mathrm{eq}}}{\partial \varepsilon}\right|_{\tau}-\left.\frac{\partial P^{\mathrm{eq}}}{\partial \tau}\right|_{\varepsilon}\right)=-\tau^{2} T^{\mathrm{eq}}\left[P^{\mathrm{eq}},-1\right] \mathrm{d}^{2} s^{\mathrm{eq}}\left[\begin{array}{c}
P^{\mathrm{eq}} \\
-1
\end{array}\right] .
\end{gathered}
$$

It was shown in [31] that the optimization problem (3.6) or (3.7) is equivalent to perform an inf-convolution between two convex functions. This result guarantees that $\mathbf{w} \mapsto s^{\text {eq }}$ is always concave and therefore that the equilibrium speed of sound $c^{\mathrm{eq}}$ satisfies $\left(c^{\mathrm{eq}}\right)^{2} \geq 0$. Let us emphasize that this condition is not sufficient for ensuring the hyperbolicity of the Euler system (2.3) (see for example the equilibrium p-system studied in $[10,11]$ ). Nevertheless, we will present in Section 4.2 additional arguments that prove that $\left(c^{\mathrm{eq}}\right)^{2}>0$ and therefore that the Euler system (2.3) is strictly hyperbolic (excluding of course the cases in which vacuum is present).

We now introduce another assumption that pertains to the thermodynamic properties of the liquid and the vapor at equilibrium (see e.g. $[3,9,28,44]$ ), namely

$$
0<y^{*}<1 \Rightarrow\left\{\tau_{l}^{*}<\tau_{v}^{*}, \varepsilon_{l}^{*}<\varepsilon_{v}^{*}, s_{l}\left(\mathbf{w}_{l}^{*}\right)<s_{v}\left(\mathbf{w}_{v}^{*}\right)\right\} .
$$

In the sequel, we shall assume that the EOS of the liquid and the vapor phases verify hypothesis (3.9). The strict inequality in assumption (3.9) means that we consider only first order phase transition. The assumed ordering of the liquid and vapor properties is satisfied for most liquid-vapor systems. 
Remark 3.1. Hypothesis (3.9) implies that the system cannot reach the critical point. Indeed at the critical point one necessarily has $\tau_{l}^{*}=\tau_{v}^{*}, \varepsilon_{l}^{*}=\varepsilon_{v}^{*}$ and $s_{l}\left(\mathbf{w}_{l}^{*}\right)=s_{v}\left(\mathbf{w}_{v}^{*}\right)$.

Assumptions (3.3) and hypothesis (3.9) provide a simple characterization of the solution of the optimization problem (3.6).

Theorem 3.2 (extremum principle). Let $\left(\mathbf{w}_{l}, \mathbf{w}_{v}, y_{l}, y_{v}\right) \mapsto \sigma$ be the off-equilibrium entropy of definition (3.4). For a given state $\mathbf{w} \in \mathcal{C}$,

(1) there is at least one state $\left(\mathbf{w}_{l}^{*}, \mathbf{w}_{v}^{*}, y_{l}^{*}, y_{v}^{*}\right) \in \mathcal{Q}(\mathbf{w})$ solution of the optimization problem (3.6);

(2) a state $\left(\mathbf{w}_{l}^{*}, \mathbf{w}_{v}^{*}, y_{l}^{*}, y_{v}^{*}\right) \in \mathcal{Q}(\mathbf{w})$ is a solution of the optimization problem (3.6) if and only if one of the following systems is satisfied:

$$
\begin{aligned}
\text { either } & \left\{\begin{array}{l}
y_{l}^{*}=1, \quad y_{v}^{*}=0, \\
s_{l}(\mathbf{w})>s_{v}(\mathbf{w}) ;
\end{array}\right. \\
\text { either } \quad\left\{\begin{array}{l}
y_{l}^{*}=0, y_{v}^{*}=1, \\
s_{l}(\mathbf{w})<s_{v}(\mathbf{w}) ;
\end{array}\right. & \left\{\begin{array}{l}
0<y_{l}^{*}, y_{v}^{*}<1, \\
\text { either } \left.\quad \frac{1}{T_{l}}, \frac{P_{l}}{T_{l}}, \frac{g_{l}}{T_{l}}\right)\left(\mathbf{w}_{l}^{*}\right)=\left(\frac{1}{T_{v}}, \frac{P_{v}}{T_{v}}, \frac{g_{v}}{T_{v}}\right)\left(\mathbf{w}_{v}^{*}\right) .
\end{array}\right.
\end{aligned}
$$

The state $\left(\mathbf{w}_{l}^{*}, \mathbf{w}_{v}^{*}, y_{l}^{*}, y_{v}^{*}\right)$ will then be referred to as an equilibrium state.

When $0<y_{l}^{*}<1$ (then $0<y_{v}^{*}<1$ ), we will say that the equilibrium state $\mathbf{w}$ is a saturated state.

(3) if $\mathbf{w} \in \mathcal{C}$ is a saturated state then there is a unique solution of the optimization problem (3.6).

(4) if $\mathbf{w} \in \mathcal{C}$ is not a saturated state, i.e. $y_{v}^{*}=1$ (resp. $y_{l}^{*}=1$ ) then $\mathbf{w}_{v}^{*}\left(\right.$ resp. $\mathbf{w}_{l}^{*}$ ) defines an unique solution for (3.4) up to the arbitrary choice of $\mathbf{w}_{l}^{*}$ (resp. $\left.\mathbf{w}_{v}^{*}\right)$ for the vanished phase.

Proof. See $[4,18,29]$.

\section{Equilibrium EOS: CONSTRUCtion}

In the present section we show that the optimization procedure involved in (3.6) reads as a direct geometrical construction of the concave hull of the function $\mathbf{w} \mapsto \max \left\{s_{l}(\mathbf{w}), s_{v}(\mathbf{w})\right\}$. Moreover, this interpretation will provide us a new equivalent definition for the saturated states that boils down to a single nonlinear scalar equation. This approach has been exploited in $[10,11,18,19,21,34]$ within a numerical simulation framework.

\subsection{Properties of the equilibrium EOS}

Following $[9-11,18,34]$ we recall a first geometrical result that characterizes the solution of (3.6).

Proposition 4.1 (bitangent plane). For $\alpha=l, v$ let $\mathcal{S}_{\alpha}$ be the surface defined by the graph of $\mathbf{w} \mapsto s_{\alpha}$ in the $(\mathbf{w}, s)$ space. Given a thermodynamical state $\mathbf{w} \in \mathcal{C}$, if $\left(\mathbf{w}_{l}^{*}, \mathbf{w}_{v}^{*}, y_{l}^{*}, y_{v}^{*}\right) \in \mathcal{Q}(\mathbf{w})$ maximizes $\left(\mathbf{w}_{l}, \mathbf{w}_{v}, y_{l}, y_{v}\right) \mapsto \sigma$ and if $0<y_{l}^{*}<1$ (equivalently $0<y_{v}^{*}<1$ ), then there exists a unique plane, called "bitangent plane", tangent to the surface $\mathcal{S}_{l}$ at the point $\left(\mathbf{w}_{l}^{*}, s_{l}^{*} \stackrel{\text { def }}{=} s_{l}\left(\mathbf{w}_{l}^{*}\right)\right)$ and to the surface $\mathcal{S}_{v}$ at the point $\left(\mathbf{w}_{v}^{*}, s_{v}^{*} \stackrel{\text { def }}{=} s_{v}\left(\mathbf{w}_{v}^{*}\right)\right)$.

Proof. For $\alpha=l, v$, let $\mathcal{P}_{\alpha}$ be the tangent plane to the surface $\mathcal{S}_{\alpha}$ at the point $\left(\mathbf{w}_{\alpha}^{*}, s_{\alpha}^{*}\right)$. We have to prove that the plane $\mathcal{P}_{l}$ and the plane $\mathcal{P}_{v}$ are the same. This boils down to show that $\mathcal{P}_{l}$ and $\mathcal{P}_{v}$ are parallels and that $\mathcal{P}_{l} \cap \mathcal{P}_{v} \neq \emptyset$. Because of $(3.1)$, the equation of $\mathcal{P}_{\alpha}$ reads $s=\left(\frac{P_{\alpha}}{T_{\alpha}}\left(\mathbf{w}_{\alpha}^{*}\right)\right) \tau+\left(\frac{1}{T_{\alpha}}\left(\mathbf{w}_{\alpha}^{*}\right)\right) \varepsilon+\frac{g_{\alpha}}{T_{\alpha}}\left(\mathbf{w}_{\alpha}^{*}\right)$. Thus $\mathcal{P}_{l}$ and $\mathcal{P}_{v}$ are parallels if and only if $P_{l}\left(\mathbf{w}_{l}^{*}\right)=P_{v}\left(\mathbf{w}_{v}^{*}\right)$ and $T_{l}\left(\mathbf{w}_{l}^{*}\right)=T_{v}\left(\mathbf{w}_{v}^{*}\right)$. Moreover, $\frac{g_{l}}{T_{l}}\left(\mathbf{w}_{l}^{*}\right)=\frac{g_{v}}{g_{v}}\left(\mathbf{w}_{v}^{*}\right)$ is equivalent to $\mathbf{w}=(0,0) \in \mathcal{P}_{l} \cap \mathcal{P}_{v} \neq \emptyset$. 
Consequently solving (3.6) leads either to $y_{l}^{*} \in\{0,1\}$ either to determine the bitangent plane to $\mathcal{S}_{l}$ and $\mathcal{S}_{v}$. We now recall a second classic result (see $[9,32]$ ) that connects the optimization problem (3.6) and the bitangent plane construction of Proposition 4.1. An alternative equivalent way, based on the inf-convolution, for constructing the equilibrium entropy $\mathbf{w} \mapsto s^{\text {eq }}$ is presented in [30].

Proposition 4.2 (concave hull). Let $\mathcal{S}$ be the graph of the equilibrium entropy $\mathbf{w} \mapsto s^{\text {eq }}$ in the (w, s) space. Then

(1) the surface $\mathcal{S}$ is the concave hull of the set

$$
\left\{(\mathbf{w}, s) \in \mathcal{C} \times \mathbb{R} \mid s \leq \max \left[s_{l}(\mathbf{w}), s_{v}(\mathbf{w})\right]\right\} ;
$$

(2) for every saturated state $\mathbf{w} \in \mathcal{C}$, the surface $\mathcal{S}$ contains a segment $\mathfrak{r}(\mathbf{w})$ passing through the point $\left(\mathbf{w}, s^{\mathrm{eq}}(\mathbf{w})\right)$. Along this segment the pressure $P^{\mathrm{eq}}$, the temperature $T^{\mathrm{eq}}$ and the chemical potential $g^{\mathrm{eq}}$ are constant.

In general $\mathbf{w} \mapsto s^{\text {eq }}$ is $\mathrm{C}^{1}$ but not $\mathrm{C}^{2}[5]$. In the following, we assume that it is piecewise $\mathrm{C}^{2}$ in the sense that the set of all saturated states (defined by (3.12)) is a $\mathrm{C}^{2}$ manifold with a boundary which is a $\mathrm{C}^{1}$ closed loop curve.

Theorem 4.3 (existence and uniqueness of the segment $\mathfrak{r}(\mathbf{w})$ ). For $\alpha=l, v$, let $\mathcal{S}_{\alpha}$ be the graph of the phasic entropy $\mathbf{w}_{\alpha} \mapsto s_{\alpha}$ in the $(\mathbf{w}, s)$ space and let $\mathbf{w} \in \mathcal{C}$ be a saturated state (defined in Thm. 3.2). Then there exists $a$ unique couple of points

$$
\begin{aligned}
& \mathbf{M}_{l}^{*} \stackrel{\text { def }}{=}\left(\mathbf{w}_{l}^{*}, s_{l}^{*} \stackrel{\text { def }}{=} s_{l}\left(\mathbf{w}_{l}^{*}\right)\right) \in \mathcal{S}_{l}, \\
& \mathbf{M}_{v}^{*} \stackrel{\text { def }}{=}\left(\mathbf{w}_{v}^{*}, s_{v}^{*} \stackrel{\text { def }}{=} s_{v}\left(\mathbf{w}_{v}^{*}\right)\right) \in \mathcal{S}_{v},
\end{aligned}
$$

such that the point $\mathbf{M} \stackrel{\text { def }}{=}\left(\mathbf{w}, s^{\mathrm{eq}}(\mathbf{w})\right)$ belongs to the line segment

$$
\mathfrak{r}(\mathbf{w}) \stackrel{\text { def }}{=}\left(\mathbf{M}_{l}^{*}, \mathbf{M}_{v}^{*}\right)=\left\{y \mathbf{M}_{l}^{*}+(1-y) \mathbf{M}_{v}^{*} \mid y \in[0,1]\right\} .
$$

Proof. The existence of the segment $\mathfrak{r}(\mathbf{w})$ follows from Proposition 4.2. We prove uniqueness: each $s_{\alpha}$ is strictly concave and increasing with respect to the variables $\tau_{\alpha}$ and $\varepsilon_{\alpha}$. Then the mapping $\left(\mathbf{w}_{l}, \mathbf{w}_{v}\right) \mapsto\left(P^{\mathrm{eq}}, T^{\mathrm{eq}}\right)$ is one-to-one. If there is another segment $\tilde{\mathfrak{r}}(\mathbf{w})=\left(\left(\tilde{\mathbf{w}}_{l}^{*}, \tilde{s}_{l}^{*}\right),\left(\tilde{\mathbf{w}}_{v}^{*}, \tilde{s}_{v}^{*}\right)\right)$ such that $\left(\mathbf{w}, s^{\text {eq }}(\mathbf{w})\right) \in \mathfrak{r}(\mathbf{w}) \cap \tilde{\mathfrak{r}}(\mathbf{w})$, since $\left(P^{\mathrm{eq}}, T^{\mathrm{eq}}\right)$ are constant along $\mathfrak{r}(\mathbf{w})$ and along $\tilde{\mathfrak{r}}(\mathbf{w})$, we have $\mathbf{w}_{\alpha}^{*}=\tilde{\mathbf{w}}_{\alpha}^{*}$ and consequently $\mathfrak{r}(\mathbf{w})=\tilde{\mathfrak{r}}(\mathbf{w})$.

\subsection{Phase-change equation}

We now build a so-called "phase-change equation" which is our key ingredient in the computation of the equilibrium entropy.

Consider a fixed couple $\mathbf{w} \stackrel{\text { def }}{=}(\tau, \varepsilon)$. Let $\left(\mathbf{w}_{l}^{*}, \mathbf{w}_{v}^{*}, y_{l}^{*}, y_{v}^{*}\right) \in \mathcal{Q}(\mathbf{w})$ be the maximizer of $\sigma$ that corresponds to a saturated state, i.e. $0<y^{*}<1$. In this case, by Theorem $3.2\left(\tau_{l}^{*}, \varepsilon_{l}^{*}, \tau_{v}^{*}, \varepsilon_{v}^{*}, y^{*}\right)$ is also the unique solution of the system (4.1)-(4.2):

$$
\left\{\begin{array}{l}
\tau=y \tau_{l}+(1-y) \tau_{v}, \\
\varepsilon=y \varepsilon_{l}+(1-y) \varepsilon_{v}, \\
P_{l}\left(\tau_{l}, \varepsilon_{l}\right)=P_{v}\left(\tau_{v}, \varepsilon_{v}\right), \\
T_{l}\left(\tau_{l}, \varepsilon_{l}\right)=T_{v}\left(\tau_{v}, \varepsilon_{v}\right), \\
g_{l}\left(\tau_{l}, \varepsilon_{l}\right)=g_{v}\left(\tau_{v}, \varepsilon_{v}\right), \\
y \in(0,1) .
\end{array}\right.
$$


Introducing the common values $(P, T)$ of the pressure and temperature, relations (4.1c)-(4.1d) allow to obtain the following equivalent system

$$
\left\{\begin{array}{c}
\tau=y \tau_{l}(P, T)+(1-y) \tau_{v}(P, T), \\
\varepsilon=y \varepsilon_{l}(P, T)+(1-y) \varepsilon_{v}(P, T), \\
g_{l}(P, T)=g_{v}(P, T), \\
y \in(0,1)
\end{array}\right.
$$

where the unknows are $(T, P, y)$. If we know $P$ and $T$, we can compute $y$ by

$$
y(P, T)=\frac{\tau-\tau_{v}(P, T)}{\tau_{l}(P, T)-\tau_{v}(P, T)}
$$

and eliminate $y$ in system (4.3). So, solving system (4.3) is equivalent to solve

$$
\left\{\begin{array}{l}
\frac{\tau-\tau_{v}(P, T)}{\tau_{l}(P, T)-\tau_{v}(P, T)}=\frac{\varepsilon-\varepsilon_{v}(P, T)}{\varepsilon_{l}(P, T)-\varepsilon_{v}(P, T)}, \\
g_{l}(P, T)=g_{v}(P, T) .
\end{array}\right.
$$

The equation (4.6b) defines the well-known coexistence curve $P \mapsto T^{\mathrm{sat}}(P)[9,28]$. In full generality, the solution of $(4.6 \mathrm{~b})$ is multivalued. Here we consider the physically relevant branch. If we note classically

$$
\tau_{\alpha}^{\text {sat }}(P) \stackrel{\text { def }}{=} \tau_{\alpha}\left(P, T^{\text {sat }}(P)\right), \quad \varepsilon_{\alpha}^{\text {sat }}(P) \stackrel{\text { def }}{=} \varepsilon_{\alpha}\left(P, T^{\text {sat }}(P)\right), \quad \alpha=l, v,
$$

then, given $(\tau, \varepsilon) \in \mathcal{C}$, solving (4.6a)-(4.6b) is equivalent to seeking $P$ as the solution of the following scalar nonlinear equation

$$
\frac{\tau-\tau_{v}^{\mathrm{sat}}(P)}{\tau_{l}^{\mathrm{sat}}(P)-\tau_{v}^{\mathrm{sat}}(P)}=\frac{\varepsilon-\varepsilon_{v}^{\mathrm{sat}}(P)}{\varepsilon_{l}^{\mathrm{sat}}(P)-\varepsilon_{v}^{\mathrm{sat}}(P)} .
$$

In the sequel, the equation (4.7) will be also referred to as the "phase-change equation" (a similar equation was proposed in [49]).

If we note $P^{*}$ the solution of the phase-change equation (4.7), then the solution $\left(\tau_{l}^{*}, \varepsilon_{l}^{*}, \tau_{v}^{*}, \varepsilon_{v}^{*}, y^{*}\right)$ of the system (4.1) reads

$$
\begin{aligned}
\tau_{l}^{*} & =\tau_{l}^{\mathrm{sat}}\left(P^{*}\right), \\
\varepsilon_{l}^{*} & =\varepsilon_{l}^{\mathrm{sat}}\left(P^{*}\right), \\
\tau_{v}^{*} & =\tau_{v}^{\mathrm{sat}}\left(P^{*}\right), \\
\varepsilon_{v}^{*} & =\varepsilon_{v}^{\mathrm{sat}}\left(P^{*}\right), \\
y^{*} & =y\left(P^{*}, T^{\mathrm{sat}}\left(P^{*}\right)\right) .
\end{aligned}
$$

Remark 4.4. In some cases it can be most useful to compute $T \mapsto P^{\mathrm{sat}}(T)$ instead of $P \mapsto T^{\mathrm{sat}}(P)$. The strategy proposed above is the same except that we replace $P$ by $T, P^{*}$ by $T^{*}$ and $T^{\mathrm{sat}}(P)$ by $P^{\mathrm{sat}}(T)$.

We can now summarize the construction of the equilibrium entropy $s^{\text {eq }}$ in the following definition.

Definition 4.5 (equilibrium entropy construction). The overall procedure for computing the equilibrium EOS lies in the following alternative: for all $\mathbf{w} \in \mathcal{C}$, 
(1) if there exists a solution to $(4.7)$, denoted $P^{*}$, and if $0<y^{*}<1\left(\right.$ where $\left.y^{*} \stackrel{\text { def }}{=} y\left(P^{*}, T^{\text {sat }}\left(P^{*}\right)\right)\right)$ then $\mathbf{w}$ is a saturated state and we set

$$
\begin{aligned}
y^{\mathrm{eq}}(\mathbf{w}) & =y^{*} \\
z^{\mathrm{eq}}(\mathbf{w}) & =y^{*} \tau_{l}^{*} / \tau \\
\psi^{\mathrm{eq}}(\mathbf{w}) & =y^{*} \varepsilon_{l}^{*} / \varepsilon \\
s^{\mathrm{eq}}(\mathbf{w}) & =y^{*} s_{l}\left(\mathbf{w}_{l}^{*}\right)+\left(1-y^{*}\right) s_{v}\left(\mathbf{w}_{v}^{*}\right), \\
P^{\mathrm{eq}}(\mathbf{w}) & =P_{l}\left(\mathbf{w}_{l}^{*}\right)=P_{v}\left(\mathbf{w}_{v}^{*}\right),
\end{aligned}
$$

where $\tau_{l}^{*} \stackrel{\text { def }}{=} \tau_{l}\left(P^{*}, T^{\mathrm{sat}}\left(P^{*}\right)\right), \varepsilon_{l}^{*} \stackrel{\text { def }}{=} \varepsilon_{l}\left(P^{*}, T^{\mathrm{sat}}\left(P^{*}\right)\right), \tau_{v}^{*} \stackrel{\text { def }}{=} \tau_{v}\left(P^{*}, T^{\mathrm{sat}}\left(P^{*}\right)\right)$ and $\varepsilon_{v}^{*} \stackrel{\text { def }}{=} \varepsilon_{v}\left(P^{*}, T^{\mathrm{sat}}\left(P^{*}\right)\right) ;$

(2) otherwise, if $(4.7)$ has no solution or if the corresponding $y^{*} \stackrel{\text { def }}{=} y\left(P^{*}, T^{\text {sat }}\left(P^{*}\right)\right)$ is outside the range $(0,1)$, (2a) if $s_{l}(\mathbf{w})>s_{v}(\mathbf{w})$ then $\mathbf{w}$ is a liquid state, therefore we set

$$
\begin{aligned}
y^{\mathrm{eq}}(\mathbf{w}) & =1, \\
z^{\mathrm{eq}}(\mathbf{w}) & =1, \\
\psi^{\mathrm{eq}}(\mathbf{w}) & =1, \\
s^{\mathrm{eq}}(\mathbf{w}) & =s_{l}(\mathbf{w}), \\
P^{\mathrm{eq}}(\mathbf{w}) & =P_{l}(\mathbf{w}) ;
\end{aligned}
$$

$(2 \mathrm{~b})$ if $s_{l}(\mathbf{w})<s_{v}(\mathbf{w})$ then $\mathbf{w}$ is a vapor state, therefore we set

$$
\begin{aligned}
y^{\mathrm{eq}}(\mathbf{w}) & =0, \\
z^{\mathrm{eq}}(\mathbf{w}) & =0, \\
\psi^{\mathrm{eq}}(\mathbf{w}) & =0, \\
s^{\mathrm{eq}}(\mathbf{w}) & =s_{v}(\mathbf{w}), \\
P^{\mathrm{eq}}(\mathbf{w}) & =P_{v}(\mathbf{w}) .
\end{aligned}
$$

\subsection{Example of equilibrium states and phase-change equation for two stiffened gas laws}

We present in this section the detailed construction of the phase-change equation (4.7) when both phases are modeled by a stiffened gas EOS. Let us first recall a few properties of this particular state law. The complete form of the stiffened gas EOS reads

$$
(\tau, \varepsilon) \mapsto s=c_{v} \ln (\varepsilon-q-\pi \tau)+c_{v}(\bar{\gamma}-1) \ln \tau+m
$$

where parameters $c_{v}>0, \bar{\gamma}>1, \pi>0, q$ and $m$ are constants describing thermodynamical properties of the fluid. Remark that the domain of definition of $s$ is not the usual cone $\mathcal{C}$ but rather the set $\tau>0$ and $\varepsilon-q-\pi \tau>0$. The case of a perfect gas EOS is recovered by setting $\pi=0$ and $q=0$.

The classic definitions (3.1) provide the following expressions for the temperature $T$, the pressure $P$ and the Gibbs potential $g$ :

$$
\begin{aligned}
& (\tau, \varepsilon) \mapsto T=\frac{\varepsilon-q-\pi \tau}{c_{v}} \\
& (\tau, \varepsilon) \mapsto P=\frac{\varepsilon-q-\pi \tau}{\tau}(\bar{\gamma}-1)-\pi \\
& (\tau, \varepsilon) \mapsto g=q+(\varepsilon-q-\pi \tau)\left(\bar{\gamma}-\frac{m}{c_{v}}-\ln \left((\varepsilon-q-\pi \tau) \tau^{(\bar{\gamma}-1)}\right)\right) .
\end{aligned}
$$


The Grüneisen coefficient $\Gamma$ and the square of the speed of sound $c$ are given by

$$
\begin{aligned}
& \Gamma \stackrel{\text { def }}{=}-\tau T\left(P s_{\varepsilon \varepsilon}-s_{\varepsilon \tau}\right)=\bar{\gamma}-1>0, \\
& c^{2} \stackrel{\text { def }}{=} \tau^{2}\left(\left.P \frac{\partial P}{\partial \varepsilon}\right|_{\tau}-\left.\frac{\partial P}{\partial \tau}\right|_{\varepsilon}\right)=\bar{\gamma}(\bar{\gamma}-1)(\varepsilon-q-\pi \tau)=\bar{\gamma}(P+\pi) \tau=\bar{\gamma} \Gamma c_{v} T>0 .
\end{aligned}
$$

By a simple change of variables we also have

$$
\begin{aligned}
& (P, T) \mapsto s=c_{v} \bar{\gamma} \ln T-c_{v} \Gamma \ln (P+\pi)+m+c_{v} \bar{\gamma} \ln c_{v}+c_{v} \Gamma \ln \Gamma \\
& (P, T) \mapsto g=c_{v} T\left(\bar{\gamma}-\ln \left(\left(c_{v} T\right)^{\bar{\gamma}}\left(\frac{\Gamma}{P+\pi}\right)\right)\right)-T m+q, \\
& (P, T) \mapsto \varepsilon=c_{v} T \frac{P+\pi \bar{\gamma}}{P+\pi}+q, \\
& (P, T) \mapsto \tau=c_{v}(\bar{\gamma}-1) \frac{T}{P+\pi} .
\end{aligned}
$$

For the sake of simplicity we note $q^{\prime} \stackrel{\text { def }}{=} m+c_{v} \bar{\gamma} \ln c_{v}+c_{v} \Gamma \ln \Gamma$ as in $[39,50]$.

For all $\mathbf{w} \in \mathcal{C}$ the value $s^{\mathrm{eq}}(\mathbf{w})$ is computed by solving numerically the phase-change equation (4.7) according to Definition 4.5. In order to obtain this equation we have to compute the four functions

$$
\tau_{\alpha}^{\text {sat }}(P) \stackrel{\text { def }}{=} \tau_{\alpha}\left(P, T^{\mathrm{sat}}(P)\right), \quad \varepsilon_{\alpha}^{\mathrm{sat}}(P) \stackrel{\text { def }}{=} \varepsilon_{\alpha}\left(P, T^{\mathrm{sat}}(P)\right), \quad \alpha=l, v .
$$

To achieve this task, we compute the coexistence curve $P \mapsto T^{\text {sat }}$ by solving equation (4.6b), namely

$$
g_{l}(P, T)=g_{v}(P, T) .
$$

If it happens that the solution is multivalued, we discard the non-physical branch of solution.

With the stiffened gas EOS's, two cases have to be considered separately:

- Case I: $q_{l}=q_{v}$. If and $c_{v_{l}} \bar{\gamma}_{l} \neq c_{v_{v}} \bar{\gamma}_{v}$, solving (4.6b) we obtain

$$
P \mapsto T^{\mathrm{sat}}(P)=A\left[\left(P+\pi_{l}\right)^{c_{v_{l}} \Gamma_{l}}\left(P+\pi_{v}\right)^{-c_{v_{v}} \Gamma_{v}}\right]^{1 / B}
$$

where

$$
A \stackrel{\text { def }}{=} \exp \left(1-\frac{q_{l}^{\prime}-q_{v}^{\prime}}{B}\right)>0, \quad B \stackrel{\text { def }}{=} c_{v_{l}} \bar{\gamma}_{l}-c_{v_{v}} \bar{\gamma}_{v}
$$

Let us note that for two-perfect gas we recover the usual linear dependence between $T^{\text {sat }}$ and $P$. We recall that hypothesis (3.9) implies some restrictions on the coefficients. An example of a stiffened gas that verifies all hypothesis of the previous sections for pressures greater than $6.7 \times 10^{4} \mathrm{~Pa}$ is shown in Table 1 and Figure 1. If $c_{v_{l}} \bar{\gamma}_{l}=c_{v_{v}} \bar{\gamma}_{v}$, we obtain a degenerate case where $P^{\text {sat }}$ is a constant defined by

$$
\frac{\left(P^{\mathrm{sat}}+\pi_{l}\right)^{c_{v_{l}} \Gamma_{l}}}{\left(P^{\mathrm{sat}}+\pi_{v}\right)^{c_{v_{v}} \Gamma_{v}}}=\exp \left(q_{l}^{\prime}-q_{v}^{\prime}\right)
$$

- Case II: $q_{l} \neq q_{v}$. We were not able to find any explicit expression for the coexistence curve. Therefore it is difficult to process the phase-change equation (4.7) for determining explicitly the equilibrium states. This case appears in [39] for the approximation of the states laws of water and dodecane. We have proposed in [19] and in Chapter 6 of [18] one possible mean for overcoming this difficulty: the key idea is to compute $P \mapsto \widehat{T}^{\mathrm{sat}}(P)$ as a simple and convenient approximation of the function $P \mapsto T^{\mathrm{sat}}(P)$. To achieve this task, 
TABLE 1. Example of parameters for two stiffened gas law that provides an explicit expression for the coexistence curve. These values are used in the tests of Section 8. This choice guarantees that the volume and the internal energy of the liquid are lower than the volume and the internal energy of the vapor while heaving a speed of sound not too large (which implies a not too strict CFL condition for the computational tests).

\begin{tabular}{c|ccccc}
\hline Phase & $c_{v}(\mathrm{~J} /(\mathrm{kg} \mathrm{K}))$ & $\bar{\gamma}$ & $\pi(\mathrm{Pa})$ & $q(\mathrm{~J} / \mathrm{kg})$ & $q^{\prime}(\mathrm{J} /(\mathrm{kg} \mathrm{K}))$ \\
\hline Liquid & 800 & 1.4 & $10^{5}$ & 0 & 1000 \\
Vapor & 1100 & 1.3 & 0 & 0 & 0 \\
\hline
\end{tabular}

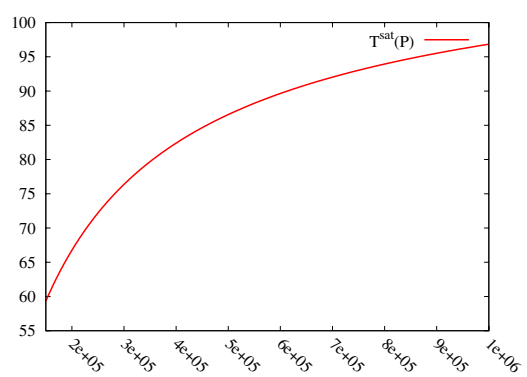

(a) $P \mapsto T^{\mathrm{sat}}(P)$

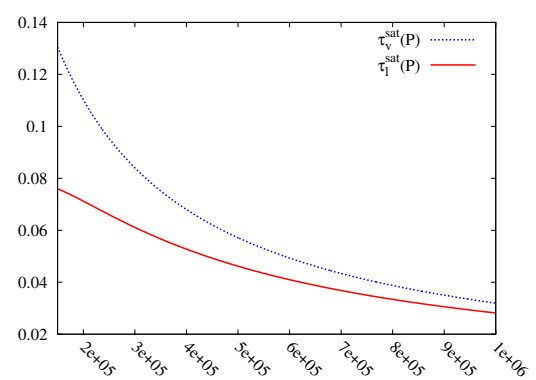

(b) $P \mapsto \tau_{l}^{\text {sat }}(P)$ (bottom) and $P \mapsto$ $\tau_{v}^{\text {sat }}(P)($ top)

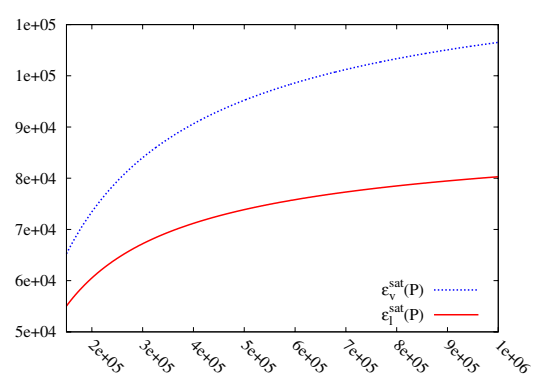

(c) $P \mapsto \varepsilon_{l}^{\text {sat }}(P)$ (bottom) and $P \mapsto$ $\varepsilon_{v}^{\text {sat }}(P)($ top $)$

Figure 1. Temperature and phasic volumes and internal energies at saturation as functions of the pressure for the stiffened gas law of Table 1.

first we discretize an interval of pressure $\left[P_{\min }, P_{\max }\right]$ and we compute (numerically but with an arbitrary precision) $\widehat{T}^{\text {sat }}$ at each point of the discretization as the solution of equation (4.6b). Thus we obtain a set of points $\mathscr{A}=\left(P_{i}, T^{\mathrm{sat}}\left(P_{i}\right)\right)_{i}$. Then we can define $P \mapsto \widehat{T}^{\text {sat }}(P)$ thanks to a least square approximation of $\mathscr{A}$. Finally, instead of solving the phase-change equation (4.7) we solve the following approximate phase-change equation:

$$
\frac{\tau-\tau_{v}\left(P, \widehat{T}^{\mathrm{sat}}(P)\right)}{\tau_{l}\left(P, \widehat{T}^{\mathrm{sat}}(P)\right)-\tau_{v}\left(P, \widehat{T}^{\mathrm{sat}}(P)\right)}=\frac{\varepsilon-\varepsilon_{v}\left(P, \widehat{T}^{\mathrm{sat}}(P)\right)}{\varepsilon_{l}\left(P, \widehat{T}^{\mathrm{sat}}(P)\right)-\varepsilon_{v}\left(P, \widehat{T}^{\mathrm{sat}}(P)\right)} .
$$

This approximation is reasonable since, in the end, the phase-change equation (exact or approximate) is always solved numerically. We already tested this approach for numerical examples in $[18,19,21]$.

Remark 4.6 (tabulated data). The case of more general EOS, including non-analytic forms of the EOS, like tabulated data, is addressed in [20,21] and Chapter 7 of [18]. Again the main idea is to build an approximate phase-change equation. In order to obtain this approximate equation, we need to evaluate the four functions

$$
\tau_{\alpha}^{\text {sat }}(P) \stackrel{\text { def }}{=} \tau_{\alpha}\left(P, T^{\mathrm{sat}}(P)\right), \quad \varepsilon_{\alpha}^{\mathrm{sat}}(P) \stackrel{\text { def }}{=} \varepsilon_{\alpha}\left(P, T^{\mathrm{sat}}(P)\right), \quad \alpha=l, v .
$$

We consider a set of experimental data values $\tau_{\alpha}^{\text {sat }}\left(P_{i}\right)$ and $\varepsilon_{\alpha}^{\text {sat }}\left(P_{i}\right)$ for a discrete set of pressure values $P_{i} \in$ $\left[P_{\min }, P_{\max }\right]$ (see e.g. [41] for an example of tabulated EOS). Then, we can define

$$
\widehat{\tau}_{\alpha}^{\mathrm{sat}}(P), \quad \widehat{\varepsilon}_{\alpha}^{\mathrm{sat}}(P) \quad \alpha=l, v, \quad \text { for } P \in\left[P_{\min }, P_{\max }\right],
$$

thanks to least square approximations. Finally, instead of solving the phase-change equation (4.7) we solve the following approximate equation

$$
\frac{\tau-\widehat{\tau}_{v}^{\mathrm{sat}}(P)}{\widehat{\tau}_{l}^{\mathrm{sat}}(P)-\widehat{\tau}_{v}^{\mathrm{sat}}(P)}=\frac{\varepsilon-\widehat{\varepsilon}_{v}^{\mathrm{sat}}(P)}{\widehat{\varepsilon}_{l}^{\mathrm{sat}}(P)-\widehat{\varepsilon}_{v}^{\mathrm{sat}}(P)} .
$$

A similar construction of equilibrium EOS is proposed in [30] too. 


\section{Properties of the Equilibrium Phase transition Model}

In this section we recall two properties of the convective part of the system (2.3), which corresponds to the Euler system equipped with the liquid-vapor thermodynamical equilibrium EOS, pertaining to the wellposedness of the system. For the sake of completeness, proofs of these theorems are provided in Appendix 9 at page 1050 and in Appendix 9 at page 1052 (these results are announced in [2]).

The first theorem is a technical result that provides informations about the first and second derivatives of the equilibrium EOS $\mathbf{w} \mapsto s^{\mathrm{eq}}$.

Theorem 5.1. Let $\mathbf{w} \in \mathcal{C}$ be a saturated state (defined in Thm. 3.2) and let $\mathfrak{r}(\mathbf{w}) \stackrel{\text { def }}{=}\left(\mathbf{M}_{l}^{*}, \mathbf{M}_{v}^{*}\right)$ be the segment associated with $\mathbf{w}$ (the segment $\mathfrak{r}(\mathbf{w})$ and points $\mathbf{M}_{\alpha}^{*}$ are defined in Thm. 4.3). Then, for every point $\mathbf{M} \stackrel{\text { def }}{=}\left(\mathbf{w}, s^{\mathrm{eq}}(\mathbf{w})\right)$ of $\mathfrak{r}(\mathbf{w})$, we have:

(A) the entries of the Hessian matrix $\mathrm{d}^{2} s^{\mathrm{eq}}$, evaluated at $\mathbf{w}$, verify

$$
\begin{gathered}
s_{\tau \varepsilon}^{\mathrm{eq}}\left(\varepsilon_{l}^{*}-\varepsilon_{v}^{*}\right)+s_{\tau \tau}^{\mathrm{eq}}\left(\tau_{l}^{*}-\tau_{v}^{*}\right)=0, \\
s_{\varepsilon \varepsilon}^{\mathrm{eq}}\left(\varepsilon_{l}^{*}-\varepsilon_{v}^{*}\right)+s_{\tau \varepsilon}^{\mathrm{eq}}\left(\tau_{l}^{*}-\tau_{v}^{*}\right)=0, \\
s_{\tau \tau}^{\mathrm{eq}} s_{\varepsilon \varepsilon}^{\mathrm{eq}}=\left(s_{\tau \varepsilon}^{\mathrm{eq}}\right)^{2} \\
s_{\tau \tau}^{\mathrm{eq}}=\left(\frac{\varepsilon_{l}^{*}-\varepsilon_{v}^{*}}{\tau_{l}^{*}-\tau_{v}^{*}}\right)^{2} s_{\varepsilon \varepsilon}^{\mathrm{eq}} ; \\
s_{\tau \tau}^{\mathrm{eq}}<0, \\
s_{\varepsilon \varepsilon}^{\mathrm{eq}}<0, \\
s_{\tau \varepsilon}^{\mathrm{eq}}>0 ;
\end{gathered}
$$

(B) the pressure $P^{\mathrm{eq}}$, evaluated at $\mathbf{w}$, verifies

$$
P^{\mathrm{eq}}>-\frac{\left(\varepsilon_{l}^{*}-\varepsilon_{v}^{*}\right)}{\left(\tau_{l}^{*}-\tau_{v}^{*}\right)}
$$

Theorem 5.1 shed light on some important properties of the Hessian matrix of the equilibrium entropy $s^{\text {eq }}$ that departs from the classic pure fluid framework. Indeed, the EOS $\mathbf{w} \mapsto s^{\mathrm{eq}}$ verify the inequality (3.3c) but does not verify the inequality (3.3b). More precisely,

- for a pure state $\mathbf{w} \in \mathcal{C}, \mathrm{d}^{2} s^{\text {eq }}$ is a negative definite matrix, namely:

$$
\mathbf{v}^{T} \cdot \mathrm{d}^{2} s^{\mathrm{eq}}(\mathbf{w}) \cdot \mathbf{v}<0 \quad \forall \mathbf{v} \neq(0,0)
$$

which implies that

$$
s_{\tau \tau}^{\mathrm{eq}}(\mathbf{w})<0, \quad s_{\varepsilon \varepsilon}^{\mathrm{eq}}(\mathbf{w})<0, \quad s_{\tau \tau}^{\mathrm{eq}}(\mathbf{w}) s_{\varepsilon \varepsilon}^{\mathrm{eq}}(\mathbf{w})>\left(s_{\tau \varepsilon}^{\mathrm{eq}}(\mathbf{w})\right)^{2} ;
$$

- for a saturated state $\mathbf{w} \in \mathcal{C}, \mathrm{d}^{2} s^{\text {eq }}$ is no longer negative definite, it is merely nonpositive and $\mathrm{d}^{2} s^{\text {eq }} \neq\left(\begin{array}{ll}0 & 0 \\ 0 & 0\end{array}\right)$, namely:

$$
\left\{\begin{array}{l}
\mathbf{v}^{T} \cdot \mathrm{d}^{2} s^{\mathrm{eq}}(\mathbf{w}) \cdot \mathbf{v} \leq 0 \quad \forall \mathbf{v} \in \mathbb{R}^{2}, \\
\exists ! \mathbf{v}(\mathbf{w}) \neq(0,0) \quad \text { such that } \quad(\mathbf{v}(\mathbf{w}))^{T} \cdot \mathrm{d}^{2} s^{\mathrm{eq}}(\mathbf{w}) \cdot \mathbf{v}(\mathbf{w})=0 .
\end{array}\right.
$$

This implies that $s_{\tau \tau}^{\mathrm{eq}}(\mathbf{w})<0, s_{\varepsilon \varepsilon}^{\mathrm{eq}}(\mathbf{w})<0, s_{\tau \tau}^{\mathrm{eq}}(\mathbf{w}) s_{\varepsilon \varepsilon}^{\mathrm{eq}}(\mathbf{w})=\left(s_{\tau \varepsilon}^{\mathrm{eq}}(\mathbf{w})\right)^{2}$ and moreover we have $s_{\tau \varepsilon}^{\mathrm{eq}}(\mathbf{w})>0$.

Theorem 5.1 allows us to prove that the Euler system equipped with the equilibrium EOS is strictly hyperbolic. This property is important for the well-posedness of the system and its numerical resolution. 
Theorem 5.2 (hyperbolicity). For smooth solutions, the compressible Euler system equipped with the liquidvapor equilibrium $E O S \mathbf{w} \mapsto s^{\mathrm{eq}}$ reads

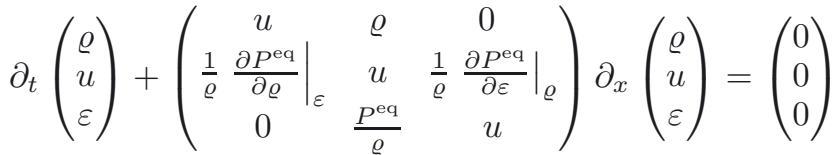

$$
\begin{aligned}
& \text { with } \varrho=\frac{1}{\tau}, \quad P^{\mathrm{eq}}=\frac{s_{\tau}^{\mathrm{eq}}}{s_{\varepsilon}^{\mathrm{eq}}} \text {. }
\end{aligned}
$$

Then, for all $\mathbf{w} \in \mathcal{C}$, the square of the speed of sound $c^{\mathrm{eq}}$, defined by (3.2a), is strictly positive and therefore $c^{\mathrm{eq}}$, defined by (3.8), is real and the system (5.4) is strictly hyperbolic.

Remark 5.3. As a corollary of our Theorem 5.2, the MTT-equilibrium system defined in [10] or the Euler system provided with the EOS defined in $[4,29,31,34,40,47,55]$ are strictly hyperbolic.

Remark 5.4. We emphasize that the positivity of the speed of sound does not seem totally trivial. First, the function $\mathbf{w} \mapsto s^{\mathrm{eq}}$ is concave (but not strictly concave) and its Hessian matrix is not negative definite. Concavity provides that $c^{2} \geq 0$, but we do not have a priori informations that ensures that $c^{2} \neq 0$. Second, for some systems as in $[10,11,26]$ the loss of strict concavity of the entropy indeed leads to a zero speed of sound: in this case hyperbolicity is lost because the system eigenvalues are real but the Jacobian matrix of the flux is no longer diagonalizable. This situation leads to ill-posed problems (see for example [42], p. 362) where uniqueness is lost within the classical class of entropy solutions.

Remark 5.5. Theorem 5.1 implies that: (1) the flux function in the Euler equations is only piecewise regular; (2) the functions $\mathbf{w} \mapsto\left(P^{\mathrm{eq}}, T^{\mathrm{eq}}\right)(\mathbf{w})$ are continuous and piecewise $\mathrm{C}^{1}$ regular: their derivatives are discontinuous when the fluid changes from a pure phase state $y \in\{0,1\}$ to a saturated state $y \in(0,1)$; (3) the speed of sound $\mathbf{w} \mapsto c^{\text {eq }}$ is piecewise continuous: it is discontinuous along the saturation curve, namely across $y \in\{0,1\}$ regions and $0<y<1$ regions.

\section{RELAXATION APPROACH}

The definition of the equilibrium entropy $s^{\mathrm{eq}}$ as the maximizer of the mixture entropy $\sigma$ suggests to read the convective part of the system (2.3), which consists of the Euler equations closed by the EOS $\mathbf{w} \mapsto s^{\mathrm{eq}}$, as the limit of a relaxed augmented Euler system equipped with the $\operatorname{EOS}(y, z, \psi, \mathbf{w}) \mapsto \sigma$ (see for example $[7,12,13,43]$ ). This procedures helps to break the implicit definition of the equilibrium EOS and enables to use of simple discretization strategies. For example, in $[29,31]$ the authors proposed to consider the system

$$
\left\{\begin{array}{l}
\partial_{t} \mathbf{U}+\operatorname{div}[\mathbf{F}(\mathbf{U}, y, z, \psi)]=\mathbf{0}, \\
\partial_{t} y+\mathbf{u} \cdot \operatorname{grad} y=\left(y-y^{\mathrm{eq}}\right) \mu_{y}, \\
\partial_{t} z+\mathbf{u} \cdot \operatorname{grad} z=\left(z-z^{\mathrm{eq}}\right) \mu_{z}, \\
\partial_{t} \psi+\mathbf{u} \cdot \operatorname{grad} \psi=\left(\psi-\psi^{\mathrm{eq}}\right) \mu_{\psi},
\end{array}\right.
$$

with

$$
\begin{gathered}
\mathbf{F}(\mathbf{U}, y, z, \psi), \stackrel{\text { def }}{=}\left(\begin{array}{c}
\varrho \mathbf{u} \\
\varrho \mathbf{u} \otimes \mathbf{u}+P \text { Id } \\
\left.\varrho\left(\varepsilon+|\mathbf{u}|^{2} / 2\right)+P\right) \mathbf{u}
\end{array}\right), \\
P(\mathbf{w}, y, z, \psi) \stackrel{\text { def }}{=} \frac{\left.\frac{\partial \sigma}{\partial \tau}\right|_{\varepsilon, z, y, \psi}}{\left.\frac{\partial \sigma}{\partial \varepsilon}\right|_{\tau, z, y, \psi}}=\frac{\sum_{\alpha=l, v} z_{\alpha} \frac{P_{\alpha}}{T_{\alpha}}}{\sum_{\alpha=l, v} \psi_{\alpha} \frac{1}{T_{\alpha}}}
\end{gathered}
$$

and $y^{\mathrm{eq}}, z^{\mathrm{eq}}, \psi^{\mathrm{eq}}$ defined in Definition 4.5. The coefficients $\mu_{y}, \mu_{z}$ and $\mu_{\psi}$ are positive relaxation parameters. 
Formally, when $\mu_{y}, \mu_{z}, \mu_{\psi} \rightarrow+\infty$ we obtain

$$
\begin{aligned}
(y, z, \psi) & \rightarrow\left(y^{\mathrm{eq}}, z^{\mathrm{eq}}, \psi^{\mathrm{eq}}\right)(\mathbf{w}), \\
\sigma(y, z, \psi, \mathbf{w}) & \rightarrow s^{\mathrm{eq}}(\mathbf{w}), \\
\mathbf{F}(\mathbf{U}, y, z, \psi) & \rightarrow \mathbf{F}^{\mathrm{eq}}(\mathbf{w})
\end{aligned}
$$

and the solution $\mathbf{U}$ of (6.1) tends to the solution of the convective part of (2.3).

When the mass fraction satisfies $0<y<1$, we remark that it is also possible to consider the following alternative augmented system

$$
\left\{\begin{array}{l}
\partial_{t} \mathbf{U}+\operatorname{div}[\mathbf{F}(\mathbf{U}, y, z, \psi)]=\mathbf{0}, \\
\partial_{t} y+\mathbf{u} \cdot \operatorname{grad} y=\left(\frac{g_{l}}{T_{l}}-\frac{g_{v}}{T_{v}}\right) \mu_{y}, \\
\partial_{t} z+\mathbf{u} \cdot \operatorname{grad} z=\left(\frac{P_{v}}{T_{v}}-\frac{P_{l}}{T_{l}}\right) \tau \mu_{z}, \\
\partial_{t} \psi+\mathbf{u} \cdot \operatorname{grad} \psi=\left(\frac{1}{T_{l}}-\frac{1}{T_{v}}\right) \varepsilon \mu_{\psi},
\end{array}\right.
$$

and, in this case, for smooth solutions we have the following entropy evolution equation

$$
\partial_{t}(\varrho \sigma)+\operatorname{div}(\varrho \sigma \mathbf{u})=\mu_{y} \varrho\left(\frac{g_{l}}{T_{l}}-\frac{g_{v}}{T_{v}}\right)^{2}+\mu_{z}\left(\frac{P_{v}}{T_{v}}-\frac{P_{l}}{T_{l}}\right)^{2}+\mu_{\psi} \varrho \varepsilon\left(\frac{1}{T_{l}}-\frac{1}{T_{v}}\right)^{2} \geq 0 .
$$

In the regime $\mu_{y}, \mu_{z}, \mu_{\psi} \rightarrow+\infty$, one retrieves the solution of the convective part of $(2.3)$ for $y \in(0,1)$.

In order to approximate the solution of the convective part of the system $(2.3)$, following similar lines as $[10,18]$ we consider another augmented system based on the five-equation system with the isothermal closure presented in $[1,36]$. Using (6.3) instead of (6.1) allows us to re-use the code developed in $[1,36]$ and to modify it in a non-intrusive manner thanks to an additional module that computes the projection onto the equilibrium states. The system reads

$$
\left\{\begin{array}{l}
\partial_{t} \mathbf{U}+\operatorname{div}[\mathbf{G}(\mathbf{U}, y, z)]=\mathbf{0} \\
\partial_{t} y+\mathbf{u} \cdot \operatorname{grad} y=\left(y-y^{\mathrm{eq}}\right) \mu_{y} \\
\partial_{t} z+\mathbf{u} \cdot \operatorname{grad} z=\left(z-z^{\mathrm{eq}}\right) \mu_{z}
\end{array}\right.
$$

where

$$
\mathbf{G}(\mathbf{U}, y, z) \stackrel{\text { def }}{=}\left(\begin{array}{c}
\varrho \mathbf{u} \\
\varrho \mathbf{u} \otimes \mathbf{u}+\Pi \mathrm{Id} \\
\left.\varrho\left(\varepsilon+|\mathbf{u}|^{2} / 2\right)+\Pi\right) \mathbf{u}
\end{array}\right), \quad \Pi(\mathbf{w}, y, z) \stackrel{\text { def }}{=} \sum_{\alpha=l, v} z_{\alpha} P_{\alpha}, \quad T_{l}=T_{v} .
$$

The solution of (6.3) formally tends to the solution of the convective part of (2.3) in the limit regime $\mu_{y}, \mu_{z} \rightarrow$ $+\infty$.

Remark 6.1. The system (6.3) can be considered as a partial equilibrium of (6.1) in the formal limit $\mu_{\psi} \rightarrow+\infty$. In this case one enforces $\psi=\psi^{\mathrm{eq}}(\mathbf{w})$, which is equivalent to $T_{l}=T_{v}$ for $y \in(0,1)$ and implies $P\left(\mathbf{w}, y, z, \psi^{\mathrm{eq}}(\mathbf{w})\right)=\Pi(\mathbf{w}, y, z)$.

Remark 6.2. The non-dissipative structure of both systems (6.1) and (6.3) can be derived thanks to a least action principles (see $[10,11,18]$ ).

\section{NuMERICAL SCHEME}

We present in this section the numerical strategy we implemented in order to solve the equilibrium system (2.3) with heat diffusion, gravity and surface tension effects. Following the analysis of Section 6 , we chose a relaxation 
approach that consists in approximating the solution of the following system

$$
\left\{\begin{array}{l}
\partial_{t} \mathbf{U}+\operatorname{div}[\mathbf{G}(\mathbf{U}, \varrho y, z)]=\mathbf{S}_{\text {tension }}(\mathbf{U}, z)+\mathbf{S}_{\text {gravity }}(\mathbf{U})-\operatorname{div}\left[\mathbf{Q}_{\text {heat }}(\mathbf{U}, z)\right] \\
\partial_{t}(\varrho y)+\operatorname{div}(\varrho y \mathbf{u})=\varrho\left(y-y^{\mathrm{eq}}\right) \mu_{y} \\
\partial_{t} z+\mathbf{u} \cdot \operatorname{grad} z=\left(z-z^{\mathrm{eq}}\right) \mu_{z}
\end{array}\right.
$$

in the limit regime $\mu_{y}, \mu_{z} \rightarrow+\infty$.

Let $(\mathbf{U}, \varrho y, z)^{n}$ be the discretized state variable at time $t=t^{n}$. The overall numerical solver is composed of the following steps:

Step I. Convection. Update $(\mathbf{U}, \varrho y, z)^{n}$ to $(\mathbf{U}, \varrho y, z)^{n+I}$ by solving the convective terms of the system (7.1), i.e. $\mathbf{S}_{\text {tension }}(\mathbf{U}, z)=\mathbf{0}, \mathbf{S}_{\text {gravity }}(\mathbf{U})=\mathbf{0}, \mathbf{Q}_{\text {heat }}(\mathbf{U}, z)=\mathbf{0}, \mu_{y}=0$ and $\mu_{z}=0$. In our numerical tests, this step is achieved thanks to the second-order explicit finite volume Roe-type solver proposed in $[1,36]$. For stability purpose the time step is constrained by a CFL condition (see $[1,36]$ for details);

Step II. Capillarity effects. Update $(\mathbf{U}, \varrho y, z)^{n+I}$ to $(\mathbf{U}, \varrho y, z)^{n+I I}$ by solving $\partial_{t} \mathbf{U}=\mathbf{S}_{\text {tension }}(\mathbf{U}, z)$ thanks to the MAC algorithm proposed in [8];

Step III. Gravity effects. Update $(\mathbf{U}, \varrho y, z)^{n+I I}$ to $(\mathbf{U}, \varrho y, z)^{n+I I I}$ by integrating $\partial_{t} \mathbf{U}=\mathbf{S}_{\text {gravity }}(\mathbf{U})$ with an explicit centered discretization;

Step IV. Thermal diffusion. Update $(\mathbf{U}, \varrho y, z)^{n+I I I}$ to $(\mathbf{U}, \varrho y, z)^{n+I V}$ by solving $\partial_{t} \mathbf{U}=-\operatorname{div}\left[\mathbf{Q}_{\text {heat }}(\mathbf{U}, z)\right]$ with a standard implicit finite volume scheme;

Step V. Projection onto the equilibrium states. Update $(\mathbf{U}, \varrho y, z)^{n+I V}$ to $(\mathbf{U}, \varrho y, z)^{n+1}$ by computing the formal limit $\mu_{y}, \mu_{z} \rightarrow+\infty$. This boils down to set

$$
\begin{aligned}
\varrho^{n+1} & =\varrho^{n+I V}, \\
\varepsilon^{n+1} & =\varepsilon^{n+I V}, \\
\mathbf{u}^{n+1} & =\mathbf{u}^{n+I V}, \\
y^{n+1} & =y^{\mathrm{eq}}\left(1 / \varrho^{n+1}, \varepsilon^{n+1}\right), \\
z^{n+1} & = \begin{cases}0, & \text { if } y^{n+1}=0, \\
y^{n+1} \varrho^{n+1} \tau_{v}^{n+1}, & \text { if } 0<y^{n+1}<1, \\
1, & \text { if } y^{n+1}=1 .\end{cases}
\end{aligned}
$$

More precisely, we seek $P^{*}$ by means of a dichotomy algorithm as the solution of the phase-change equation (4.7) with $\tau=1 / \varrho^{n+1}$ and $\varepsilon=\varepsilon^{n+1}$, namely here:

$$
\frac{\frac{1}{\varrho^{n+1}}-\tau_{v}^{\mathrm{sat}}(P)}{\tau_{l}^{\mathrm{sat}}(P)-\tau_{v}^{\mathrm{sat}}(P)}=\frac{\varepsilon^{n+1}-\varepsilon_{v}^{\mathrm{sat}}(P)}{\varepsilon_{l}^{\mathrm{sat}}(P)-\varepsilon_{v}^{\mathrm{sat}}(P)} .
$$

Then, following definition 4.5 , we set

$$
\left(\begin{array}{l}
P \\
y \\
z
\end{array}\right)^{n+1}=\left(\begin{array}{l}
P \\
y \\
z
\end{array}\right)^{\text {eq }}\left(\frac{1}{\varrho^{n+1}}, \varepsilon^{n+1}\right)
$$

This completes the definition of $(\mathbf{U}, y, z)^{n+1}$.

Remark 7.1. The functions $(\tau, \varepsilon) \mapsto P^{*}$ and $(\tau, \varepsilon) \mapsto y^{*}$ can be computed once and for all prior to the start of any flow simulation. Let us note that although the pressure $P^{\text {eq }}$ is merely continuous and piecewise $\mathrm{C}^{1}$ regular (cf. Rem. 5.5), this scheme did not generate spurious oscillations in the solutions of our tests. 


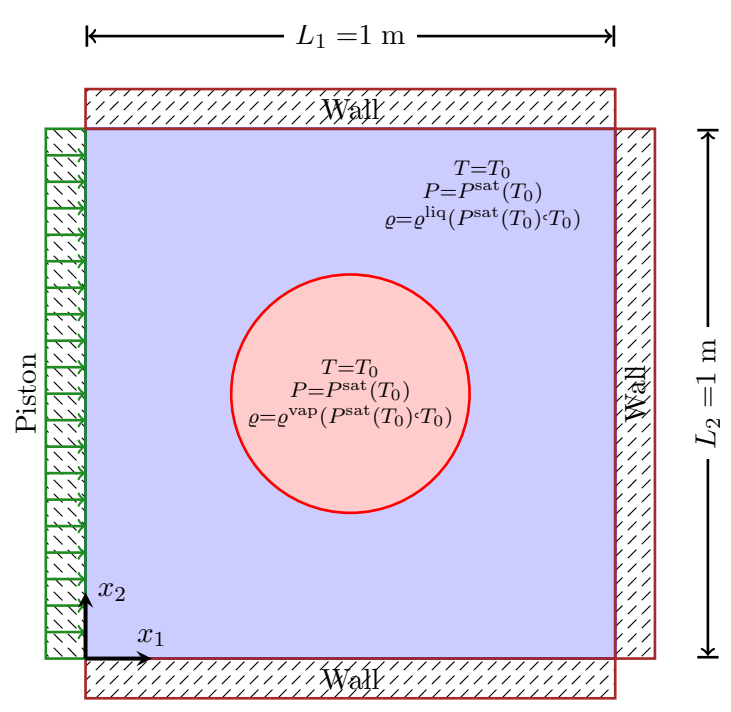

FiguRE 2. Geometry of the test "8.1. compression of vapor bubble".

\section{Numerical Simulations}

The present tests challenge the ability of our approach to reproduce cavitation phenomena and nucleation phenomena in pool boiling type conditions. As the last test involves gravity, surface tension and thermal diffusion, in order to obtain not exceedingly long calculation time, we choose to carefully fit the state laws and the physical parameters. Indeed, the CFL constraint imposed by the explicit scheme of the step I does not allow to use laws fitted on real water tabulated data: the driving phenomena for such tests are thermal diffusion and gravity and are too small compared to the speed of sound appearing in the CFL constraint. However we emphasize that there is no technical difficulty in using our approach with real fluid laws, even tabulated data (see for example the tests that reproduce cavitation phenomena in $[18,19])$. Theses improvements will be presented in forthcoming papers.

\subsection{Compression of vapor bubble}

Our model is tested here on the case of vapor bubble compression (see Fig. 2). We consider a $1 \mathrm{~m}$ sidelength 2D square domain discretized over a $400 \times 400$ cell mesh. Vapor bubble is surrounded by liquid phase in the center of the domain. The radius is initially $r=0.15 \mathrm{~m}$. Both EOS used are "Stiffened Gas" type whose coefficients are given in Table 1 . The initial pressure is fixed to $P_{0}=13046.85692 \mathrm{~Pa}$ and the fluid is initially at rest $(\mathbf{u}=\mathbf{0})$ in the whole domain. Both phases are supposed to be at saturation at $t=0$, therefore the densities for the liquid and the vapor are respectively $\varrho_{\text {liq }}=35.32714277 \mathrm{~kg} / \mathrm{m}^{3}, \varrho_{\text {vap }}=3.953593004 \mathrm{~kg} / \mathrm{m}^{3}$. We suppose the left boundary to be a piston. We proceed by duplicating the value of $\varrho, y, z, P$ and transverse velocity from the last cell close to the boundary into to the fictitious cell. Then we set the normal velocity to $u_{p}$. We chose here $u_{p}=1.0 \mathrm{~m} / \mathrm{s}$. Other boundary conditions are reflective walls.

Figure 3 shows the volume fraction, the mass fraction, the density, the pressure and the temperature for time varying from $t=0 \mathrm{~ms}$ to $t=100 \mathrm{~ms}$. The color field corresponds to the phases: light color around $z=y=0$ (vapor) and dark around $z=y=1$ (liquid). From the top to the bottom the following sequence can be seen: the moving piston generates a pressure wave that compresses the vapor. As the pressure increases the vapor starts to condensate and the bubble shrinks. 

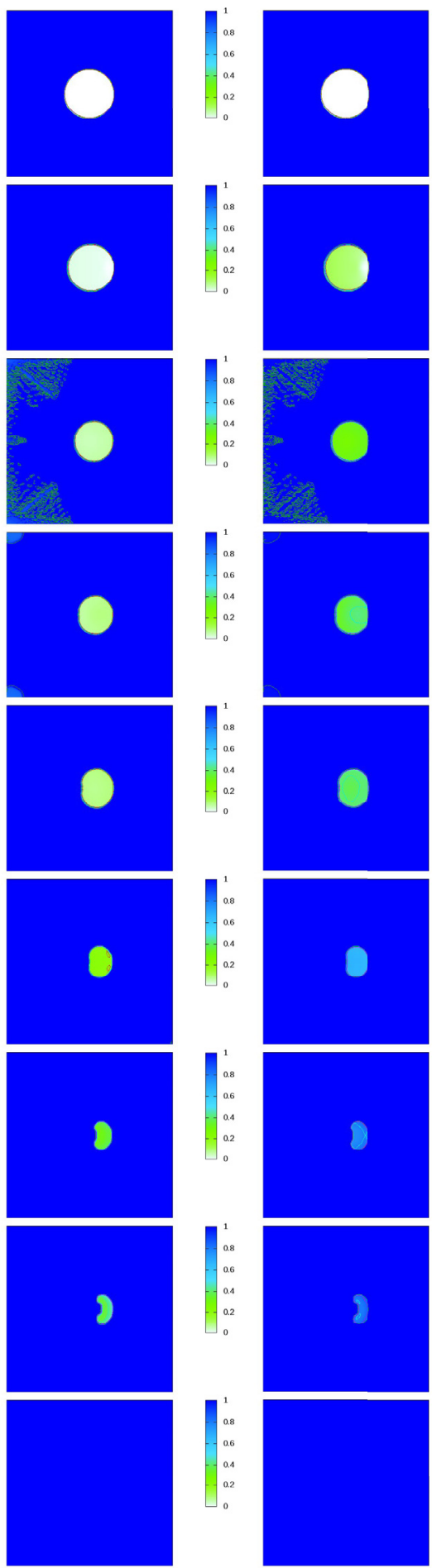
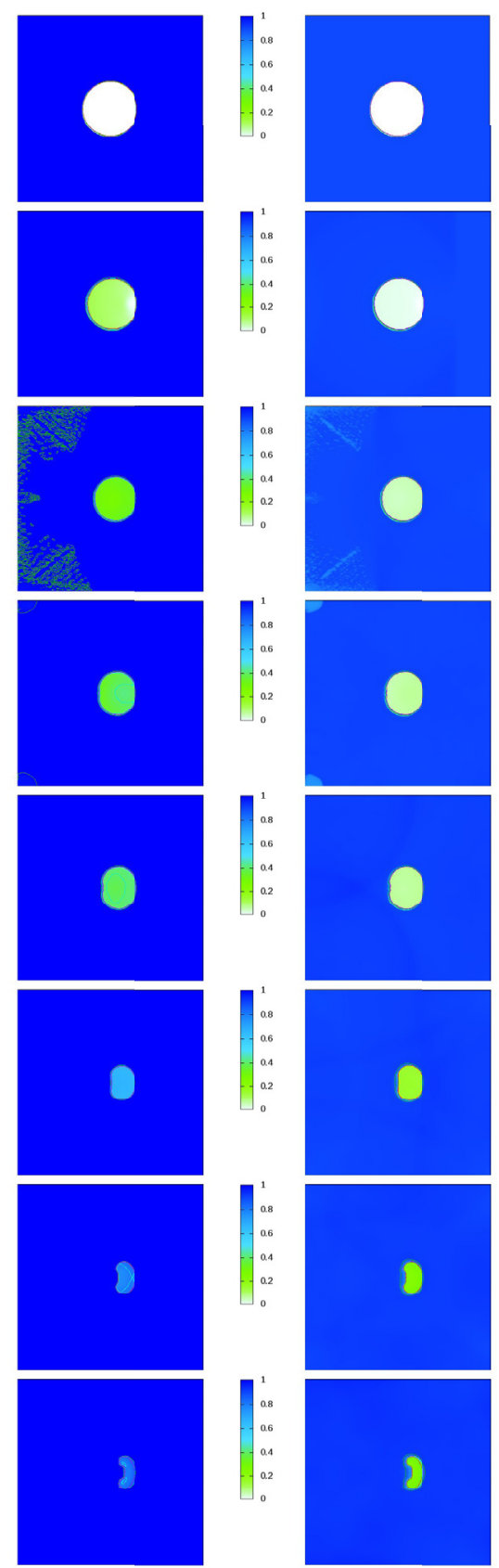

1
0.8
0.6
0.4
0.2
0
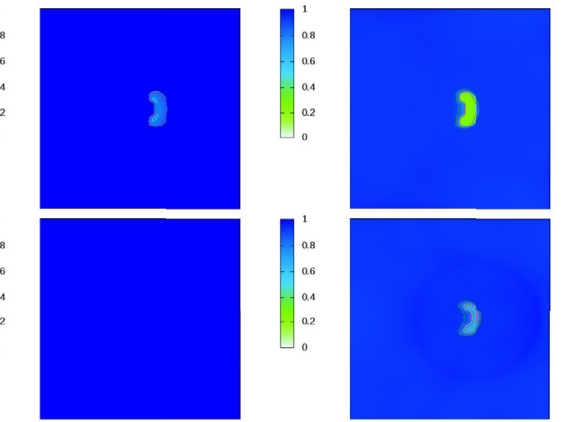
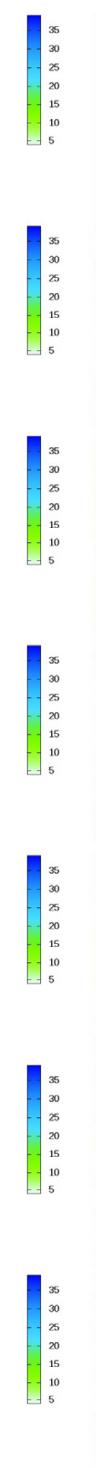

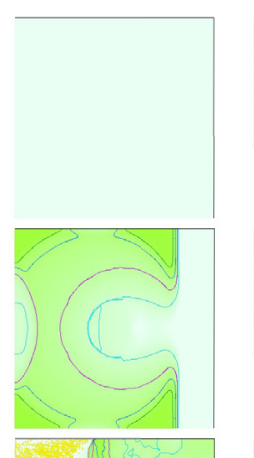

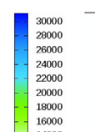
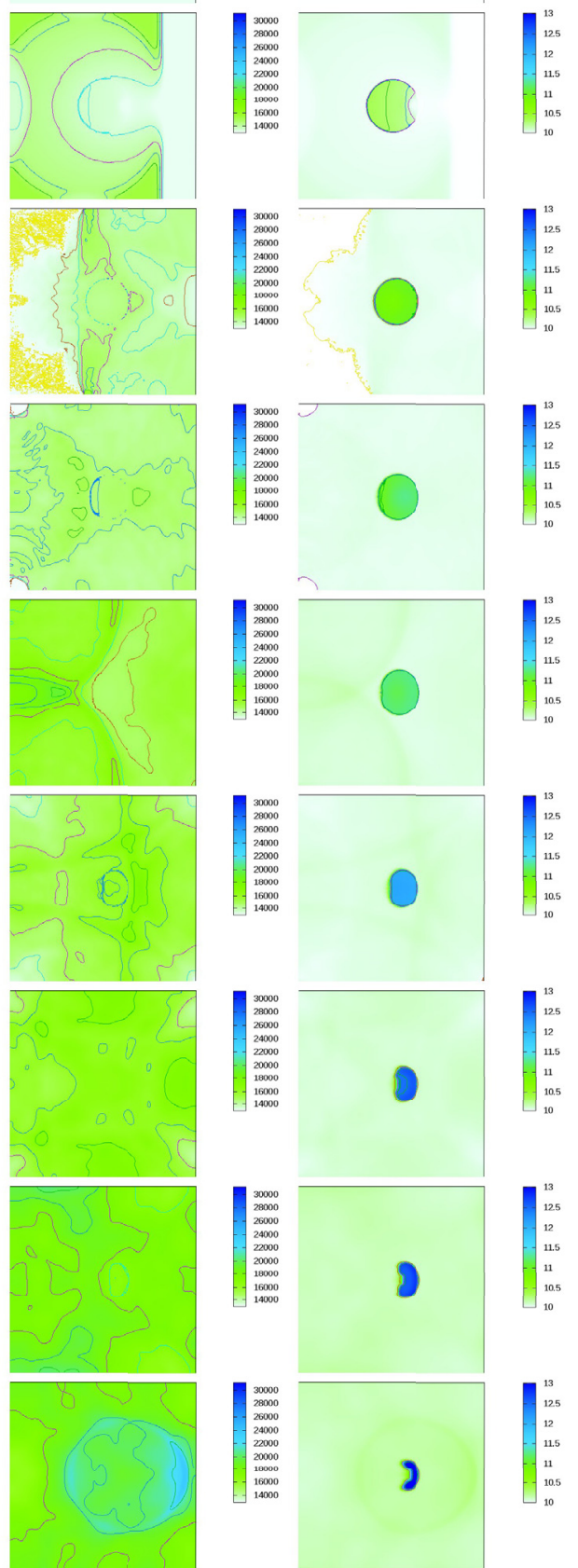

Figure 3. Time evolution of the volume fraction (first column), the mass fraction (second column), the density (third column), the pressure (fourth column) and the temperature (last column) of the test "8.1. compression of vapor bubble". 


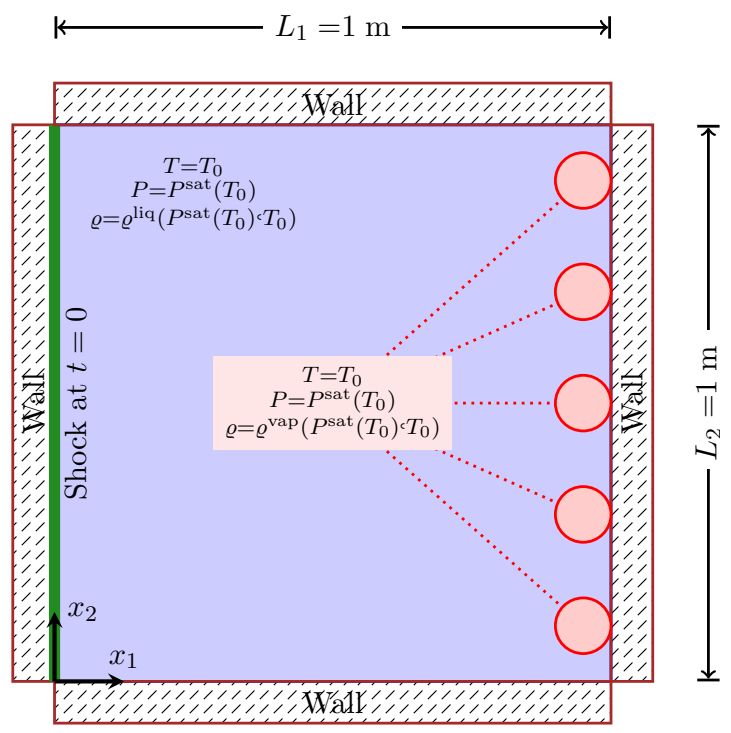

FIGURE 4. Geometry of the test "8.2. liquefaction and vaporisation caused by a shock and its bounce".

\subsection{Liquefaction and vaporisation caused by a shock and its bounce}

Our model is tested here on the case of a phase change caused by a shock and its rebounding (see Fig. 4 for the initial configuration). We consider a $1 \mathrm{~m}$ side-length 2D square domain discretized over a $200 \times 200$ cell mesh. Vapor bubbles in the right of the domain are surrounded by liquid phase. The radius of each bubble is initially $r=0.04 \mathrm{~m}$. Both EOS used are "Stiffened Gas" type whose coefficients are given in Table 1. Both phases are supposed to be at saturation at $t=0$ and the thermodynamical conditions are as in the test "compression of vapor bubble". The fluid is initially at rest $(\mathbf{u}=\mathbf{0})$ in the whole domain. To mimic an initial shock we impose a moving piston boundary condition ( $c f$. test 8.1 ) with velocity $u_{p}=100 \mathrm{~m} / \mathrm{s}$ only for the first time step. In the subsequent time step we enforce reflective wall boundary condition. Other boundary conditions are reflective walls.

Figure 5 shows the volume fraction, the mass fraction, the density, the pressure and the temperature for time varying from $t=0 \mathrm{~ms}$ to $t=100 \mathrm{~ms}$. The color field corresponds to the phases: light color around $z=y=0$ (vapor) and dark around $z=y=1$ (liquid). The shock compresses the vapor bubbles which liquefy as in the test "compression of vapor bubble". But after the bounce of the shock on the right wall, the pressure decreases and the liquid vaporizes at the original location of the bubbles.

\subsection{Bubbles nucleation, detachment and rise}

To study the ability of the model to deal with two-phase flows with phase change, we study numerically the nucleation and the motion of bubbles inside a closed box under the action of capillary, gravity and thermal flux imposed. We consider a fluid whose liquid phase and vapor phase are modeled by two stiffened gas EOSs. The thermodynamics parameters are given in Table 1 . The gravity acceleration is $\mathbf{g}=(0,-100) \mathrm{m} / \mathrm{s}^{2}$. The thermal conductivity are $\vartheta_{l}=67.8 \mathrm{~W} /(\mathrm{m} \mathrm{K})$ and $\vartheta_{v}=4.24 \mathrm{~W} /(\mathrm{m} \mathrm{K})$ and the surface tension is $\xi=7 \mathrm{~N} / \mathrm{m}$. The computational domain is a square of $1 \mathrm{~m}$ side-length initially filled with the liquid phase (see Fig. 6). The liquid is initially at rest $(\mathbf{u}=\mathbf{0})$ with uniform temperature $T_{0}=5.25 \mathrm{~K}$. The initial pressure is given by the hydrostatic pressure with a top reference pressure $P^{\mathrm{ref}}=6.8 \times 10^{3} \mathrm{~Pa}>P^{\mathrm{sat}}\left(T_{0}\right) \approx 6.73 \times 10^{3} \mathrm{~Pa}$. At the top, we keep both pressure and temperature constant at $P=P^{\mathrm{ref}}$ and $T=T^{\mathrm{ref}}=1.0 \mathrm{~K}$. At the bottom we consider 

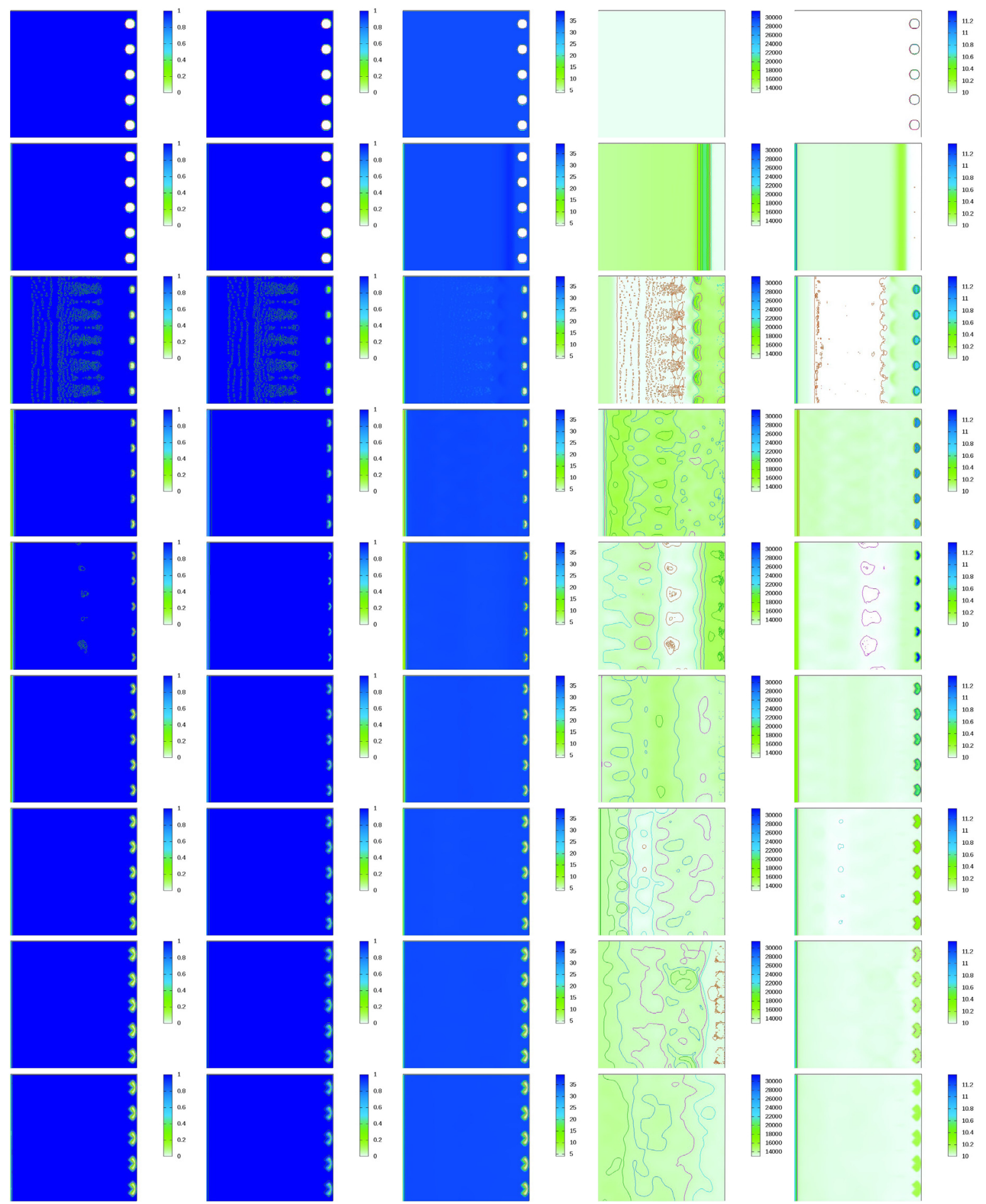

Figure 5. Time evolution of the volume fraction (first column), the mass fraction (second column), the density (third column), the pressure (fourth column) and the temperature (last column) of the test "8.2. liquefaction and vaporisation caused by a shock and its bounce". 


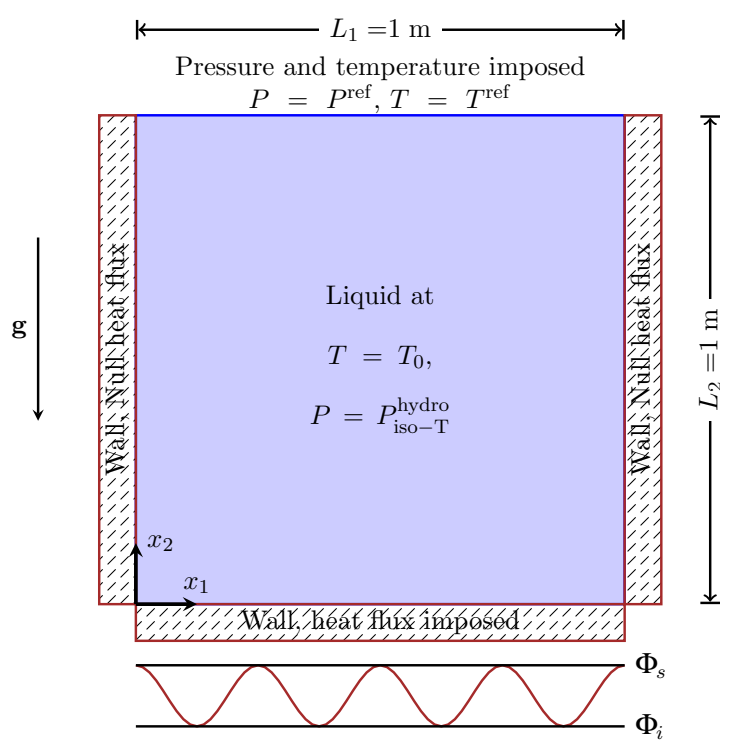

Figure 6. Geometry of the test "8.3. bubbles nucleation, detachment and rise".

wall boundary condition for the convection operator and we impose the following thermal flux profile:

$$
\Phi\left(x_{1}, x_{2}=0, t\right) \stackrel{\text { def }}{=} \frac{1}{2}\left(\left(\Phi_{s}+\Phi_{i}\right)+\left(\Phi_{s}-\Phi_{i}\right) \cos \left(4 \pi\left(x_{1}-0.5\right)\right)\right)
$$

with $\Phi_{i}=0 \mathrm{~W} / \mathrm{m}^{2}$ and $\Phi_{s}=20 \mathrm{Wm}^{2}$. We impose a homogeneous Neumann boundary conditions for both convection and thermal diffusion operators at the left and right boundary. For our test, the domain is discretized with a $100 \times 100$ cell mesh.

Figure 7 displays the results obtained for the volume fraction, the mass fraction, the density, the pressure and the temperature. We observe that nucleation of vapor bubbles occurs at the point of maximum heat flux at the bottom boundary. The bubble of vapor grows due to the phase change, then it is lifted off the boundary by gravity effects. One can notice that after the first bubble takes off, a portion of vapor remains at the bottom. As the bubble rises it reaches a region where the liquid is cooler. The vapor is then cooled by thermal diffusion and the fluid liquefies. But very quickly, the nucleation sites at the bottom starts to spread and merge at the bottom of the domain.

\section{Conclusion And Perspectives}

We investigated the simulation of dynamical liquid-vapor phase change in compressible flows. Our model considers both phase to be compressible fluids equipped with their own EOS. Local and instantaneous interphase equilibria are assumed which provides the definition of an equilibrium EOS for the two-phase medium by means of an equilibrium entropy law. We reviewed the connection between this equilibrium entropy and the concave hull of the pure phases entropy law. This allowed us to prove that even though the equilibrium entropy is not strictly concave, the resulting equilibrium system is strictly hyperbolic. The definition of the equilibrium entropy is obtained thanks to an implicit nonlinear system of equations that involves the phasic variables. Nevertheless, we showed that it is possible to reduce this system to a nonlinear scalar equation (4.7) based on the saturation law of the pure phases, which are common experimental data. We exploited this property in our numerical algorithm. A two-step relaxation strategy was used to decouple the approximation of the convection operator from the evaluation of the equilibrium EOS. While hydrodynamics is resolved in the first 

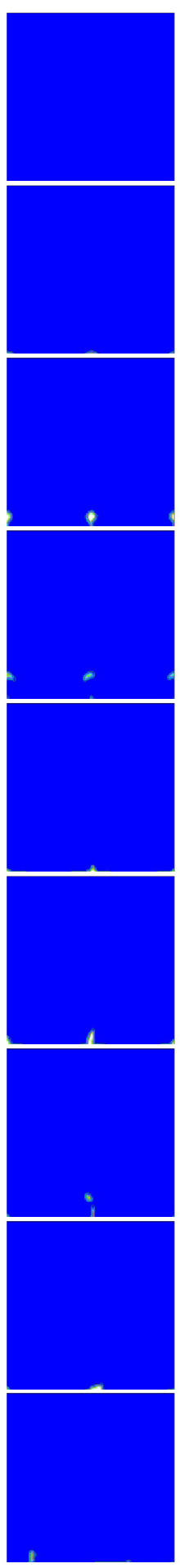
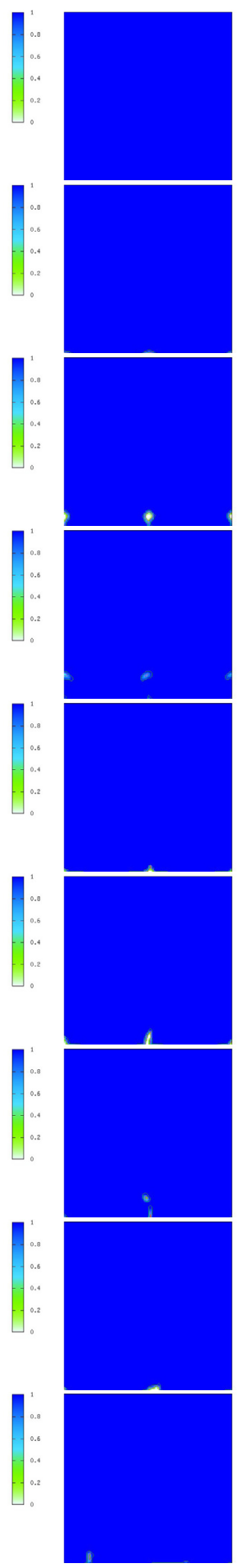
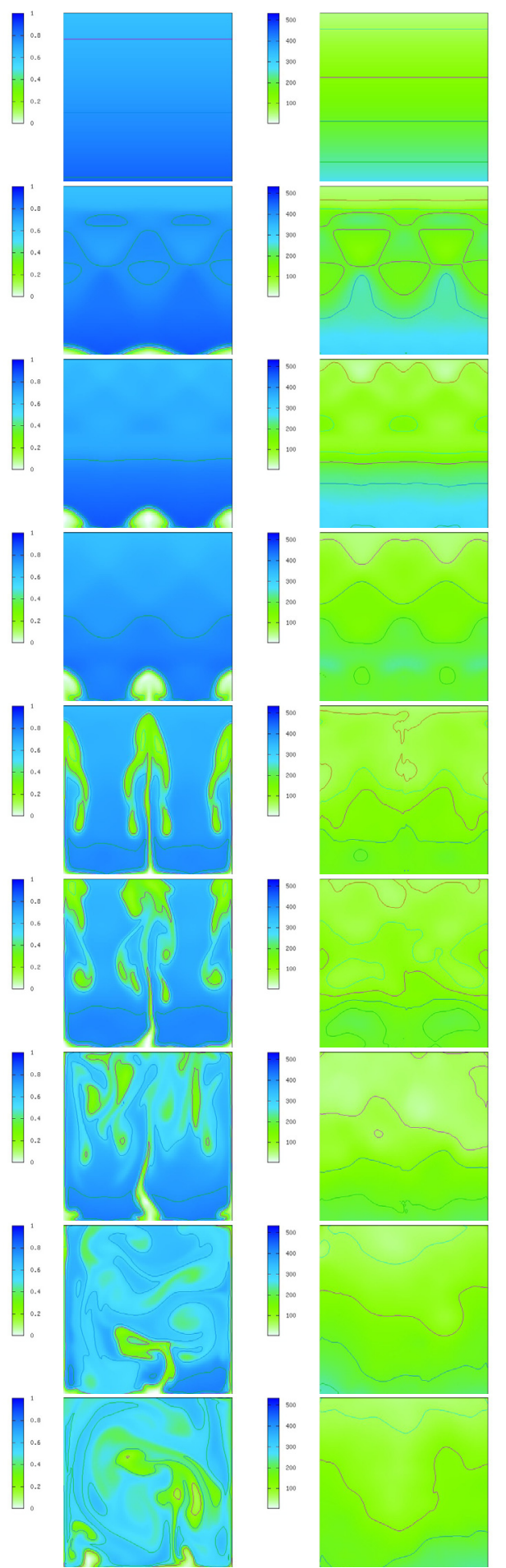
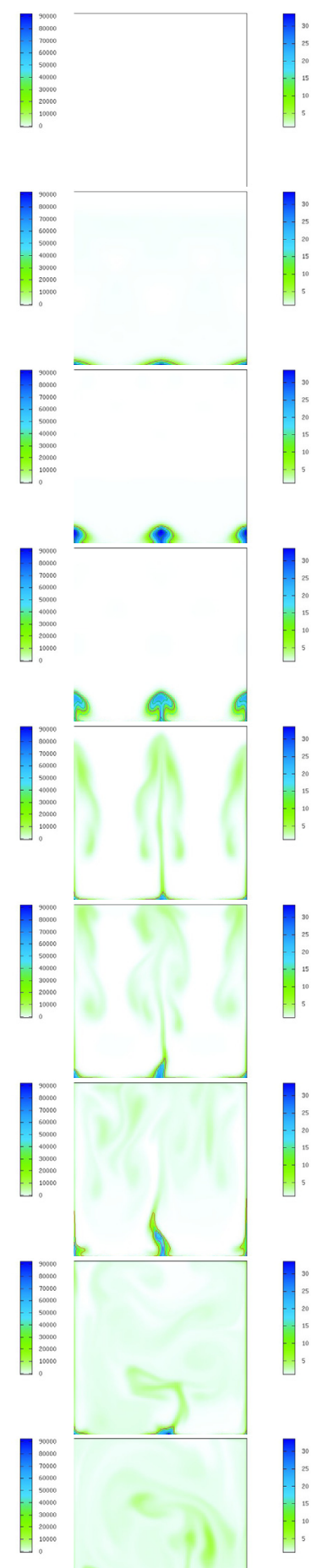

Figure 7. Time evolution of the volume fraction (first column), the mass fraction (second column), the density (third column), the pressure (fourth column) and the temperature (last column) of the test "8.3. bubbles nucleation, detachment and rise". 
step by means of a simple two-phase model, the computation of the inter-phase equilibria is confined within the last step that consists in projecting the variables onto the equilibrium states. We proposed two-dimensional numerical simulations of boiling phenomena involving Stiffened Gas EOS for the pure phases, surface tension, gravity and thermal diffusion. The results showed that the model can reproduce nucleation mechanisms, in the sense that vapor can be created in an initially pure liquid domain. Even thought theses results are only qualitative they are important toward the study of the bubble growth dynamics.

The present work can be enriched in several ways. From a modeling point of view, additional physical effects may be taken into account such as fluid viscosity and contact angle to model the transition from nucleate boiling to film boiling. From a numerical point of view, the simulation of boiling-type tests with realistic physical laws involves large time-scale processes compared to the sound velocity of the medium. This limitation may be circumvented by implementing implicit numerical methods for the convection operator.

\section{Appendix A. Proof of Theorem 5.1}

Proof of (5.1). The jump of specific volume, energy and entropy (postulated at hypothesis 3.9) implies that for every saturated state $\mathbf{w}$

$$
0<y^{*}<1, \quad \tau_{l}^{*}<\tau_{v}^{*}, \quad \varepsilon_{l}^{*}<\varepsilon_{v}^{*}, \quad s_{l}^{*} \stackrel{\text { def }}{=}\left(s_{l}\left(\mathbf{w}_{l}^{*}\right)\right)<s_{v}^{* \text { def }}=\left(s_{v}\left(\mathbf{w}_{v}^{*}\right)\right) .
$$

In this case, $\tau_{\alpha}^{*}, \varepsilon_{\alpha}^{*}$ and $y^{*}$ are such that $\mathbf{w}=y^{*} \mathbf{w}_{l}^{*}+\left(1-y^{*}\right) \mathbf{w}_{v}^{*}$ and $s^{\mathrm{eq}}(\mathbf{w})=y^{*} s_{l}^{*}+\left(1-y^{*}\right) s_{v}^{*}$.

Along the segment $\mathfrak{r}(\mathbf{w})$, the pressure $P^{\text {eq }} \stackrel{\text { def }}{=} \frac{s_{\tau}^{*}}{s_{\varepsilon}^{\text {eq }}}$ and the temperature $T^{\text {eq }} \stackrel{\frac{\text { def }}{=}}{\frac{1}{s_{\varepsilon}^{\text {eq }}}}$ are constant. Then, the directional derivatives read

$$
\begin{aligned}
& 0=\mathrm{d}\left(\frac{P^{\mathrm{eq}}}{T^{\mathrm{eq}}}\right) \cdot\left(\mathbf{w}_{l}^{*}-\mathbf{w}_{v}^{*}\right)=s_{\tau \varepsilon}^{\mathrm{eq}}\left(\varepsilon_{l}^{*}-\varepsilon_{v}^{*}\right)+s_{\tau \tau}^{\mathrm{eq}}\left(\tau_{l}^{*}-\tau_{v}^{*}\right), \\
& 0=\mathrm{d}\left(\frac{1}{T^{\mathrm{eq}}}\right) \cdot\left(\mathbf{w}_{l}^{*}-\mathbf{w}_{v}^{*}\right)=s_{\varepsilon \varepsilon}^{\mathrm{eq}}\left(\varepsilon_{l}^{*}-\varepsilon_{v}^{*}\right)+s_{\tau \varepsilon}^{\mathrm{eq}}\left(\tau_{l}^{*}-\tau_{v}^{*}\right),
\end{aligned}
$$

which proves (5.1a) and (5.1b). Then (5.1c) follows by taking the determinant of the singular Hessian matrix. Eventually (5.1d) is a simple consequence of (5.1a) and (5.1b).

Proof of (5.2). By construction $\mathbf{w} \mapsto s^{\mathrm{eq}}$ is concave. This implies that $s_{\tau \tau}^{\mathrm{eq}} \leq 0$ and $s_{\varepsilon \varepsilon}^{\mathrm{eq}} \leq 0$. However $\mathbf{w} \mapsto s^{\mathrm{eq}}$ is not strictly concave. Since we proved the inequalities $(5.1)$, it is sufficient to prove that $s_{\tau \tau}^{\mathrm{eq}}(\mathbf{w}) \neq 0$ for all saturated state $\mathbf{w}$.

We proceed by contradiction. Let $\overline{\mathbf{w}}$ be a saturated state such that $s_{\tau \tau}^{\mathrm{eq}}(\overline{\mathbf{w}})=0$. By equalities $(5.1 \mathrm{~b})-(5.1 \mathrm{c})$, the Hessian matrix is null at $\overline{\mathbf{w}}$.

We note $(\bar{P}, \bar{T}, \bar{g}) \stackrel{\text { def }}{=}\left(P_{\alpha}, T_{\alpha}, g_{\alpha}\right)(\overline{\mathbf{w}})$ for $\alpha=l, v$ (we know that $P^{\mathrm{eq}}, T^{\mathrm{eq}}$ and $g^{\mathrm{eq}}$ are constant along the segment $\mathfrak{r}(\mathbf{w}))$.

We consider a regular $\mathrm{C}^{2}$ curve inscribed on the graph $\mathcal{S}$ of $s^{\mathrm{eq}}$ and parametrized as follows

$$
t \in[-1,1] \mapsto\left(\mathbf{w}, \gamma \stackrel{\text { def }}{=} s^{\text {eq }}(\mathbf{w})\right)(t)
$$

such that $\mathbf{w}(0)=\overline{\mathbf{w}}$ and $\frac{\mathrm{d}_{\alpha}^{*}(t=0)}{\mathrm{d} t} \neq 0$ with $t \in[-1,1] \mapsto \mathbf{w}_{\alpha}^{*}(t)$ the ends of the line segment $t \in[-1,1] \mapsto$ $\mathfrak{r}(\mathbf{w}(t))$.

For the sake of simplicity we note

$$
\mathrm{d} s^{\mathrm{eq}}(\mathbf{w}(t)) \stackrel{\text { def }}{=} \frac{\mathrm{d} s^{\mathrm{eq}}}{\mathrm{d} \mathbf{w}}, \quad \mathrm{d}^{2} s^{\mathrm{eq}}(\mathbf{w}(t)) \stackrel{\text { def }}{=} \frac{\mathrm{d}^{2} s^{\mathrm{eq}}}{\mathrm{d}_{\mathbf{w}}^{2}} .
$$

We have

$$
\gamma^{\prime \prime}(t)=\frac{\mathrm{d}^{2} s^{\mathrm{eq}}}{\mathrm{d} t^{2}}=\mathrm{d} s^{\mathrm{eq}}(\mathbf{w}(t)) \frac{\mathrm{d}^{2} \mathbf{w}(t)}{\mathrm{d} t^{2}}+\frac{\mathrm{d} \mathbf{w}^{T}}{\mathrm{~d} t} \mathrm{~d}^{2} s^{\mathrm{eq}}(\mathbf{w}(t)) \frac{\mathrm{d} \mathbf{w}(t)}{\mathrm{d} t}
$$


Then, for $t=0$, we have

$$
\mathbf{w}(0)=\overline{\mathbf{w}}, \quad \mathrm{d}^{2} s^{\mathrm{eq}}(\overline{\mathbf{w}})=\left(\begin{array}{c}
00 \\
00
\end{array}\right), \quad \gamma^{\prime \prime}(0)=\mathrm{d} s^{\mathrm{eq}}(\overline{\mathbf{w}}) \frac{\mathrm{d}^{2} \mathbf{w}(0)}{\mathrm{d} t^{2}} .
$$

We now proceed to another computation of $\gamma^{\prime \prime}(0)$. By the implicit function theorem, in a vicinity of 0 , there exist $\mathrm{C}^{2}$ functions $t \mapsto y_{\alpha}^{*}$ and $t \mapsto \mathbf{w}_{\alpha}^{*}$ such that

$$
\mathbf{w}(t)=\sum_{\alpha=l, v} y_{\alpha}^{*}(t) \mathbf{w}_{\alpha}^{*}(t), \quad \gamma(t)=\sum_{\alpha=l, v} y_{\alpha}^{*}(t) s_{\alpha}^{*}\left(\mathbf{w}_{\alpha}^{*}(t)\right),
$$

where $y_{l}^{*}=y^{*}$ et $y_{v}^{*}=1-y^{*}$. We note

$$
\mathrm{d} s_{\alpha}^{*} \stackrel{\text { def }}{=} \mathrm{d} s_{\alpha}\left(\mathbf{w}_{\alpha}^{*}(t)\right) \stackrel{\text { def }}{=} \frac{\mathrm{d} s_{\alpha}}{\mathrm{d} \mathbf{w}_{\alpha}}, \quad \mathrm{d}^{2} s_{\alpha}^{*} \stackrel{\text { def }}{=} \mathrm{d}^{2} s_{\alpha}\left(\mathbf{w}_{\alpha}^{*}(t)\right) \stackrel{\text { def }}{=} \frac{\mathrm{d}^{2} s_{\alpha}^{*}}{\mathrm{~d} \mathbf{w}_{\alpha}^{2}}
$$

We have

$$
\frac{\mathrm{d}^{2} \mathbf{w}}{\mathrm{d} t^{2}}=\sum_{\alpha=l, v}\left(\frac{\mathrm{d}^{2} y_{\alpha}^{*}}{\mathrm{~d} t^{2}} \mathbf{w}_{\alpha}^{*}+2 \frac{\mathrm{d} y_{\alpha}^{*}}{\mathrm{~d} t} \frac{\mathrm{d} \mathbf{w}_{\alpha}^{*}}{\mathrm{~d} t}+y_{\alpha}^{*} \frac{\mathrm{d}^{2} \mathbf{w}_{\alpha}^{*}}{\mathrm{~d} t^{2}}\right)
$$

and

$$
\gamma^{\prime \prime}(t)=\sum_{\alpha=l, v}\left[\frac{\mathrm{d}^{2} y_{\alpha}^{*}}{\mathrm{~d} t^{2}} s_{\alpha}^{*}+2 \frac{\mathrm{d} y_{\alpha}^{*}}{\mathrm{~d} t} \mathrm{~d} s_{\alpha}^{*} \frac{\mathrm{d} \mathbf{w}_{\alpha}^{*}}{\mathrm{~d} t}+y_{\alpha}^{*} \mathrm{~d} s_{\alpha}^{*} \frac{\mathrm{d}^{2} \mathbf{w}_{\alpha}^{*}}{\mathrm{~d} t^{2}}+y_{\alpha}^{*}\left(\frac{\mathrm{d} \mathbf{w}_{\alpha}^{*}}{\mathrm{~d} t}\right)^{T} \mathrm{~d}^{2} s_{\alpha}^{*} \frac{\mathrm{d} \mathbf{w}_{\alpha}^{*}}{\mathrm{~d} t}\right],
$$

where $s_{\alpha}^{*} \stackrel{\text { def }}{=} s_{\alpha}\left(\mathbf{w}_{\alpha}^{*}\right)$. Since, by Proposition $4.2, \mathrm{~d} s_{\alpha}\left(\mathbf{w}_{\alpha}^{*}(0)\right)=\mathrm{d} s^{\mathrm{eq}}(\mathbf{w}(0))$ (namely $P_{\alpha}\left(\mathbf{w}_{\alpha}^{*}(0)\right)=P(\mathbf{w}(0))$ and $\left.T_{\alpha}\left(\mathbf{w}_{\alpha}^{*}(0)\right)=T(\mathbf{w}(0))\right)$ we obtain

$$
\sum_{\alpha=l, v}\left(2 \frac{\mathrm{d} y_{\alpha}^{*}}{\mathrm{~d} t} \mathrm{~d} s_{\alpha}^{*} \frac{\mathrm{d} \mathbf{w}_{\alpha}^{*}}{\mathrm{~d} t}+y_{\alpha}^{*} \mathrm{~d} s_{\alpha}^{*} \frac{\mathrm{d}^{2} \mathbf{w}_{\alpha}^{*}}{\mathrm{~d} t^{2}}\right)=\mathrm{d} s^{\mathrm{eq}}(\overline{\mathbf{w}}) \sum_{\alpha=l, v}\left(2 \frac{\mathrm{d} y_{\alpha}^{*}}{\mathrm{~d} t} \frac{\mathrm{d} \mathbf{w}_{\alpha}^{*}}{\mathrm{~d} t}+y_{\alpha}^{*} \frac{\mathrm{d}^{2} \mathbf{w}_{\alpha}^{*}}{\mathrm{~d} t^{2}}\right) .
$$

By relation (A.1), we have then at $t=0$

$$
\mathrm{d} s^{\mathrm{eq}}(\overline{\mathbf{w}}) \sum_{\alpha=l, v}\left(2 \frac{\mathrm{d} y_{\alpha}^{*}}{\mathrm{~d} t} \frac{\mathrm{d} \mathbf{w}_{\alpha}^{*}}{\mathrm{~d} t}+y_{\alpha}^{*} \frac{\mathrm{d}^{2} \mathbf{w}_{\alpha}^{*}}{\mathrm{~d} t^{2}}\right)=\mathrm{d} s^{\mathrm{eq}} \frac{\mathrm{d}^{2} \mathbf{w}}{\mathrm{d} t^{2}}-\sum_{\alpha=l, v} \frac{\mathrm{d}^{2} y_{\alpha}^{*}}{\mathrm{~d} t^{2}} \mathrm{~d} s_{\alpha}^{*} \mathbf{w}_{\alpha}^{*}
$$

which implies

$$
\gamma^{\prime \prime}(0)=\mathrm{d} s^{\mathrm{eq}}(\overline{\mathbf{w}}) \frac{\mathrm{d}^{2} \mathbf{w}(0)}{\mathrm{d} t^{2}}-\frac{\mathrm{d}^{2} y^{*}}{\mathrm{~d} t^{2}}\left(s_{l}^{*}-\mathrm{d} s_{l}^{*} \mathbf{w}_{l}^{*}-s_{v}^{*}+\mathrm{d} s_{v}^{*} \mathbf{w}_{v}^{*}\right)+\sum_{\alpha=l, v} y_{\alpha}^{*}\left(\frac{\mathrm{d} \mathbf{w}_{\alpha}^{*}}{\mathrm{~d} t}\right)^{T} \mathrm{~d}^{2} s_{\alpha}^{*} \frac{\mathrm{d} \mathbf{w}_{\alpha}^{*}}{\mathrm{~d} t}
$$

that reads

$$
\gamma^{\prime \prime}(0)=\mathrm{d} s^{\mathrm{eq}}(\overline{\mathbf{w}}) \frac{\mathrm{d}^{2} \mathbf{w}(0)}{\mathrm{d} t^{2}}-\frac{\mathrm{d}^{2} y^{*}}{\mathrm{~d} t^{2}}\left(\frac{g_{l}}{T_{l}}-\frac{g_{v}}{T_{v}}\right)+\sum_{\alpha=l, v} y_{\alpha}^{*}\left(\frac{\mathrm{d} \mathbf{w}_{\alpha}^{*}}{\mathrm{~d} t}\right)^{T} \mathrm{~d}^{2} s_{\alpha}^{*} \frac{\mathrm{d} \mathbf{w}_{\alpha}^{*}}{\mathrm{~d} t},
$$

as $s_{\alpha}^{*}-\mathrm{d} s_{\alpha}^{*} \mathbf{w}_{\alpha}^{*}=s_{\alpha}^{*}-\frac{P_{\alpha}}{T_{\alpha}} \tau_{\alpha}^{*}-\frac{1}{T_{\alpha}} \varepsilon_{\alpha}^{*}=\frac{g_{\alpha}}{T_{\alpha}}$.

Since $\frac{g_{\alpha}}{T_{\alpha}}=\frac{\bar{g}}{\bar{T}}$, this implies

$$
\gamma^{\prime \prime}(0)=\mathrm{d} s^{\mathrm{eq}}(\overline{\mathbf{w}}) \frac{\mathrm{d}^{2} \mathbf{w}(0)}{\mathrm{d} t^{2}}+\sum_{\alpha=l, v} y_{\alpha}^{*}\left(\frac{\mathrm{d} \mathbf{w}_{\alpha}^{*}}{\mathrm{~d} t}\right)^{T} \mathrm{~d}^{2} s_{\alpha}^{*} \frac{\mathrm{d} \mathbf{w}_{\alpha}^{*}}{\mathrm{~d} t} .
$$


Finally we have

$$
\mathrm{d} s^{\mathrm{eq}}(\overline{\mathbf{w}}) \frac{\mathrm{d}^{2} \mathbf{w}(0)}{\mathrm{d} t^{2}}=\gamma^{\prime \prime}(0)=\mathrm{d} s^{\mathrm{eq}}(\overline{\mathbf{w}}) \frac{\mathrm{d}^{2} \mathbf{w}(0)}{\mathrm{d} t^{2}}+\sum_{\alpha=l, v} y_{\alpha}^{*}\left(\frac{\mathrm{d} \mathbf{w}_{\alpha}^{*}}{\mathrm{~d} t}\right)^{T} \mathrm{~d}^{2} s_{\alpha}^{*} \frac{\mathrm{d} \mathbf{w}_{\alpha}^{*}}{\mathrm{~d} t} .
$$

Consequently, we deduce

$$
0=\sum_{\alpha=l, v} y_{\alpha}^{*}\left(\frac{\mathrm{d} \mathbf{w}_{\alpha}^{*}}{\mathrm{~d} t}\right)^{T} \mathrm{~d}^{2} s_{\alpha}^{*}\left(\frac{\mathrm{d} \mathbf{w}_{\alpha}^{*}}{\mathrm{~d} t}\right)
$$

As $\frac{\mathrm{d}_{\alpha}^{*}(t=0)}{\mathrm{d} t} \neq \mathbf{0}$ and the Hessian matrices $\mathrm{d}^{2} s_{\alpha}^{*}$ of the phasic entropies $s_{\alpha}$ are negative definite, (A.2) is impossible.

Therefore $s_{\tau \varepsilon}^{\text {eq }} \neq 0$ and $\operatorname{since~} \operatorname{sgn}\left(\varepsilon_{l}^{*}-\varepsilon_{v}^{*}\right)=\operatorname{sgn}\left(\tau_{l}^{*}-\tau_{v}^{*}\right)$, thanks to relation $(5.1 \mathrm{~b})$ we obtain the inequality

$$
s_{\tau \varepsilon}^{\mathrm{eq}}=-s_{\varepsilon \varepsilon}^{\mathrm{eq}} \frac{\varepsilon_{l}^{*}-\varepsilon_{v}^{*}}{\tau_{l}^{*}-\tau_{v}^{*}}>0 .
$$

Proof of (5.3). The inequality involving $P^{\mathrm{eq}}$ follows from the definition of the Gibbs potential. Indeed, along the segment $\mathfrak{r}(\mathbf{w})$ we have

$$
0=g_{l}-g_{v}=\left(\varepsilon_{l}^{*}-\varepsilon_{v}^{*}\right)+P^{\mathrm{eq}}\left(\tau_{l}^{*}-\tau_{v}^{*}\right)-T^{\mathrm{eq}}\left(s_{l}^{*}-s_{v}^{*}\right) .
$$

Granting the assumptions $T^{\mathrm{eq}}>0, \tau_{l}^{*}<\tau_{v}^{*}$ and $s_{l}^{*}<s_{v}^{*}$, we obtain that

$$
P^{\mathrm{eq}}=-\frac{\left(\varepsilon_{l}^{*}-\varepsilon_{v}^{*}\right)}{\left(\tau_{l}^{*}-\tau_{v}^{*}\right)}+T^{\mathrm{eq}} \frac{\left(s_{l}^{*}-s_{v}^{*}\right)}{\left(\tau_{l}^{*}-\tau_{v}^{*}\right)}>-\frac{\left(\varepsilon_{l}^{*}-\varepsilon_{v}^{*}\right)}{\left(\tau_{l}^{*}-\tau_{v}^{*}\right)}
$$

This concludes the proof.

\section{ProOf OF ThEOREM 5.2}

In one space dimension, the Jacobian matrix of the flux $\mathbf{F}^{\mathrm{eq}}$ has three eigenvalues

$$
\lambda_{1} \stackrel{\text { def }}{=} u-c^{\text {eq }}, \quad \lambda_{2} \stackrel{\text { def }}{=} u, \quad \lambda_{3} \stackrel{\text { def }}{=} u+c^{\text {eq }},
$$

with the corresponding three right eigenvectors

$$
\mathbf{r}_{1} \stackrel{\text { def }}{=}\left(\begin{array}{c}
\varrho^{2} \\
-c^{\mathrm{eq}} \varrho \\
P^{\mathrm{eq}}
\end{array}\right), \quad \mathbf{r}_{2} \stackrel{\text { def }}{=}\left(\begin{array}{c}
\left.\frac{\partial P^{\mathrm{eq}}}{\partial \varepsilon}\right|_{\varrho} \\
0 \\
-\left.\frac{\partial P^{\mathrm{eq}}}{\partial \varrho}\right|_{\varepsilon}
\end{array}\right), \quad \mathbf{r}_{3} \stackrel{\text { def }}{=}\left(\begin{array}{c}
\varrho^{2} \\
c^{\mathrm{eq}} \varrho \\
P^{\mathrm{eq}}
\end{array}\right) .
$$

Therefore, we only have to check that $\left(c^{\mathrm{eq}}\right)^{2}>0$ which immediately ensures that the three eigenvalues are distincts and the three eigenvectors are linearly independent. As $P^{\mathrm{eq}}=s_{\tau}^{\mathrm{eq}} / s_{\varepsilon}^{\mathrm{eq}}$, the square of the speed of sound verifies (3.2b), namely

$$
\left(c^{\mathrm{eq}}\right)^{2}=-\tau^{2} T^{\mathrm{eq}}\left[P^{\mathrm{eq}},-1\right]\left[\begin{array}{cc}
s_{\varepsilon \varepsilon}^{\mathrm{eq}} & s_{\tau \varepsilon}^{\mathrm{eq}} \\
s_{\varepsilon \tau}^{\mathrm{eq}} & s_{\tau \tau}^{\mathrm{eq}}
\end{array}\right]\left[\begin{array}{c}
P^{\mathrm{eq}} \\
-1
\end{array}\right] .
$$

We distinguish two cases:

Pure phase states. $(y \in\{0,1\})$ : if $\mathbf{w}$ is a pure phase state then $s^{\mathrm{eq}}(\mathbf{w}) \equiv s_{\alpha}(\mathbf{w})$ with $\alpha=l$ if $y=1$ and $\alpha=v$ if $y=0$. Therefore, using (3.3) in (A.3), we obtain that the entropy $\mathbf{w} \mapsto s^{\text {eq }}$ satisfies

$$
\left(c^{\mathrm{eq}}\right)^{2}>-\frac{\tau^{2} T^{\mathrm{eq}}}{s_{\tau \tau}^{\mathrm{eq}}}\left(P^{\mathrm{eq}} s_{\varepsilon \tau}^{\mathrm{eq}}-s_{\tau \tau}^{\mathrm{eq}}\right)^{2} \geq 0
$$


Saturated states. $(0<y<1)$ : in Theorem 5.1 we proved that if $\mathbf{w}$ is a saturated state then $\mathbf{w} \mapsto s^{\text {eq }}$ does not satisfies (3.3b). However, taking into account (5.1c) we obtain from (A.3)

$$
\left(c^{\mathrm{eq}}\right)^{2}=-\tau^{2} T^{\mathrm{eq}} \frac{1}{s_{\tau \tau}^{\mathrm{eq}}}\left(P^{\mathrm{eq}} s_{\varepsilon \tau}^{\mathrm{eq}}-s_{\tau \tau}^{\mathrm{eq}}\right)^{2},
$$

and thanks to the relation (5.1a), we finally have

$$
\left(c^{\mathrm{eq}}\right)^{2}=-\tau^{2} T^{\mathrm{eq}} \frac{\left(s_{\varepsilon \tau}^{\mathrm{eq}}\right)^{2}}{s_{\tau \tau}^{\mathrm{eq}}}\left(P^{\mathrm{eq}}+\frac{\varepsilon_{l}^{*}-\varepsilon_{v}^{*}}{\tau_{l}^{*}-\tau_{v}^{*}}\right)^{2}
$$

Thanks to the inequalities (5.2) and (5.3), this shows that $\left(c^{\mathrm{eq}}\right)^{2}>0$.

We can conclude that for all $\mathbf{w} \in \mathcal{C},\left(c^{\mathrm{eq}}(\mathbf{w})\right)^{2}$ is positive, which implies that $c^{\mathrm{eq}}(\mathbf{w})$ is real and $\neq 0$ and the system (5.4) is strictly hyperbolic.

\section{REFERENCES}

[1] G. Allaire, S. Clerc and S. Kokh, A five-equation model for the simulation of interfaces between compressible fluids. J. Comput. Phys. 181 (2002) 577-616.

[2] G. Allaire, G. Faccanoni and S. Kokh, A strictly hyperbolic equilibrium phase transition model. C. R. Acad. Sci. Paris, Sér. I 344 (2007) 135-140.

[3] K. Annamalai and I.K. Puri, Advanced thermodynamics engineering. CRC Press (2002).

[4] Th. Barberon and Ph. Helluy, Finite volume simulations of cavitating flows. Comput. Fluids 34 (2005) $832-858$.

[5] J. Benoist and J.-B. Hiriart-Urruty, What is the subdifferential of the closed convex hull of a function? SIAM J. Math. Anal. 27 (1996) 1661-1679.

[6] S. Benzoni Gavage, Stability of multi-dimensional phase transitions in a Van der Waals fluid. Nonlinear Anal. 31 (1998) 243-263.

[7] F. Bouchut, Nonlinear stability of finite volume methods for hyperbolic conservation laws and well-balanced schemes for sources. Frontiers in Mathematics. Birkhäuser Verlag, Basel (2004).

[8] J.U. Brackbill, D.B. Kothe and C. Zemach, A continuum method for modeling surface tension. J. Comput. Phys. 100 (1992) $335-354$.

[9] H.B. Callen, Thermodynamics and an introduction to thermostatistics. John Wiley \& sons, 2nd edition (1985).

[10] F. Caro, Modélisation et simulation numérique des transitions de phase liquide-vapeur. Ph.D. thesis, École Polytechnique (2004). http://www.imprimerie.polytechnique.fr/Theses/Files/caro.pdf .

[11] F. Caro, F. Coquel, D. Jamet and S. Kokh, A simple finite-volume method for compressible isothermal twophase flows simulation. International Journal on Finite Volumes (2006). http://www.latp.univ-mrs.fr/IJFVDB/ ijfv-caro-coquel-jamet-kokh.pdf.

[12] G. Chen, C.D. Levermore and T.-P. Liu, Hyperbolic conservation laws with stiff relaxation terms and entropy. Comm. Pure Appl. Math. 47 (1992) 787-830.

[13] F. Coquel and B. Perthame, Relaxation of energy and approximate Riemann solvers for general pressure laws in fluids dynamics. SIAM J. Numer. Anal. 35 (1998) 2223-2249.

[14] J.-M. Delhaye, M. Giot and M.L. Riethmuller, Thermohydraulics of two-phase systems for industrial design and nuclear engineering. Hemisphere Publishing Corporation (1981).

[15] J.-M. Delhaye, M. Giot, L. Mahias, P. Raymond and C. Rénault, Thermohydraulique des réacteurs. EDP Sciences (1998).

[16] V.K. Dhir, Boiling heat transfer. Ann. Rev. Fluid Mech. 30 (1998) 365-401.

[17] J.E. Dunn and J. Serrin, On the thermomechanics of interstitial working. Arch. Rational Mech. Anal. 88 (1985) $95-133$.

[18] G. Faccanoni, Étude d'un modèle fin de changement de phase liquide-vapeur. Contribution à l'étude de la crise d'ébullition. Ph.D. thesis, École Polytechnique, France (2008). http://pastel.paristech.org/4785/.

[19] G. Faccanoni, S. Kokh and G. Allaire, Numerical simulation with finite volume of dynamic liquid-vapor phase transition, Finite Volumes for Complex Applications V. ISTE and Wiley (2008) 391-398.

[20] G. Faccanoni, G. Allaire and S. Kokh, Modelling and numerical simulation of liquid-vapor phase transition, in Conf. Proc. of EUROTHERM-84, Seminar on Thermodynamics of Phase Changes, Namur (2009).

[21] G. Faccanoni, S. Kokh and G. Allaire, Approximation of liquid-vapor phase transition for compressible fluids with tabulated EOS. C. R. Acad. Sci. Paris Sér. I 348 (2010) 473-478.

[22] H. Fan, One phase Riemann problem and wave interactions in systems of conservation laws of mixed type. SIAM J. Math. Anal. 24 (1993) 840-865.

[23] H. Fan, Traveling waves, Riemann problems and computations of a model of the dynamics of liquid/vapor phase transitions. J. Differ. Equ. 150 (1998) 385-437. 
[24] H. Fan and M. Slemrod, The Riemann problem for systems of conservation laws of mixed type, in Conf. Proc. on Shock Induced Transitions and Phase Structure in General Media Institute of Mathematics and its Applications. Minneapolis (1990) 61-91.

[25] C. Fouillet, Généralisation à des mélanges binaires de la méthode du second gradient et application à la simulation numérique directe de l'ébullition nuclée. Ph.D. thesis, Université Paris 6 (2003).

[26] E. Godlewski and N. Seguin, The Riemann problem for a simple model of phase transition. Commun. Math. Sci. 4 (2006) $227-247$.

[27] H. Gouin, Utilization of the second gradient theory in continuum mechanics to study the motion and thermodynamics of liquid-vapor interfaces. Physicochemical Hydrodynamics - Interfacial Phenomena B 174 (1987) 667-682.

[28] W. Greiner, L. Neise and H. Stöcker, Thermodynamics and statistical mechanics. Springer (1997).

[29] Ph. Helluy, Quelques exemples de méthodes numériques récentes pour le calcul des écoulements multiphasiques. Mémoire d'habilitation à diriger des recherches (2005).

[30] Ph. Helluy and H. Mathis, Pressure laws and fast Legendre transform. Math. Models Methods Appl. Sci. to appear.

[31] Ph. Helluy and N. Seguin, Relaxation models of phase transition flows. ESAIM: M2AN 40 (2006) 331-352.

[32] J.-B. Hiriart-Urruty and C. Lemaréchal, Fundamentals of convex analysis. Grundlehren Text Editions, Springer-Verlag, Berlin (2001).

[33] D. Jamet, O. Lebaigue, N. Coutris and J.-M. Delhaye, The second gradient method for the direct numerical simulation of liquid-vapor flows with phase change. J. Comput. Phys. 169 (2001) 624-651.

[34] S. Jaouen, Étude mathématique et numérique de stabilité pour des modèles hydrodynamiques avec transition de phase. Ph.D. thesis, Université Paris 6, France (2001).

[35] S. Jin and C.D. Levermore, Numerical schemes for hyperbolic conservation laws with stiff relaxation terms. J. Comput. Phys. 126 (1996) 449-467.

[36] S. Kokh, Aspects numériques et théoriques de la modélisation des écoulements diphasiques compressibles par des méthodes de capture d'interfaces. Ph.D. thesis, Université Paris 6 (2001).

[37] D.J. Korteweg, Sur la forme que prennent les équations des mouvements des fluides si l'on tient compte des forces capillaires par des variations de densité. Arch. Néer. Sci. Exactes Sér. II 6 (1901) 1-24.

[38] P.G. LeFloch, Hyperbolic systems of conservation laws. Birkhäuser Verlag, Basel (2002).

[39] O. Le Métayer, J. Massoni and R. Saurel, Elaborating equations of state of a liquid and its vapor for two-phase flow models. Int. J. Thermal Sci. 43 (2004) 265-276.

[40] O. Le Métayer, J. Massoni and R. Saurel, Modelling evaporation fronts with reactive Riemann solvers. J. Comput. Phys. 205 (2005) 567-610.

[41] E.W. Lemmon, M.O. McLinden and D.G. Friend, Thermophysical properties of fluid systems, in WebBook de Chimie NIST, Base de Données Standard de Référence NIST Numéro 69, National Institute of Standards and Technology, edited by P.J. Linstrom and W.G. Mallard. Gaithersburg MD, 20899, http://webbook.nist.gov.

[42] R.J. LeVeque, Finite Volume methods for hyperbolic problems. Cambridge University Press, Cambridge. Appl. Math. (2002).

[43] T.P. Liu, Hyperbolic conservation laws with relaxation. Commun. Math. Phys. 108 (1987) 153-175.

[44] T. Matolcsi, On the classification of phase transitions. Z. Angew. Math. Phys. 47 (1996) 837-857.

[45] R. Menikoff and B. Plohr, The Riemann problem for fluid flow of real materials. Rev. Mod. Phys. 61 (1989) 75-130.

[46] S. Nukiyama, The maximum and minimum values of the heat $\mathrm{Q}$ transmitted from metal to boiling water under atmospheric pressure. Int. J. Heat Mass Transfer 9 (1966) 1419-1433. (English translation of the original paper published in J. Jpn Soc. Mech. Eng. 37 (1934) 367-374).

[47] F. Petitpas, E. Franquet, R. Saurel and O. Le Métayer, A relaxation-projection method for compressible flows. II. Artificial heat exchanges for multiphase shocks. J. Comput. Phys. 225 (2007) 2214-2248.

[48] P. Ruyer, Modèle de champ de phase pour l'étude de l'ébullition. Ph.D. thesis, École Polytechnique (2006). www.imprimerie. polytechnique.fr/Theses/Files/Ruyer.pdf.

[49] R. Saurel, J.-P. Cocchi and P.-B. Butlers, Numerical study of cavitation in the wake of a hypervelocity underwater projectile. J. Propuls. Power 15 (1999) 513-522.

[50] R. Saurel, F. Petitpas and R. Abgrall, Modelling phase transition in metastable liquids: application to cavitating and flashing flows. J. Fluid Mech. 607 (2008) 313-350.

[51] M. Shearer, Admissibility criteria for shock wave solutions of a system of conservation laws of mixed type. Proc. R. Soc. Edinb. 93 (1983) 133-244.

[52] M. Slemrod, Admissibility criteria for propagating phase boundaries in a van der Waals fluid. Arch. Rational Mech. Anal. 81 (1983) 301-315.

[53] L. Truskinovsky, Kinks versus shocks, in Shock induced transitions and phase structures in general media, edited by R. Fosdick et al. Springer Verlag, Berlin (1991).

[54] P. Van Carey, Liquid-vapor phase-change phenomena. Taylor and Francis (1992).

[55] A. Voß, Exact Riemann solution for the Euler equations with nonconvex and nonsmooth equation of State. Ph.D. thesis, RWTH-Aachen (2004). http://www.it-voss.com/papers/thesis-voss-030205-128-final.pdf. 